\title{
Review
}

\section{Density functional theory as a tool for the structure determination of radiation-induced bioradicals ${ }^{\dagger}$}

\author{
F. De Proft, ${ }^{1 *}$ E. Pauwels, ${ }^{2}$ P. Lahorte, ${ }^{3}$ V. Van Speybroeck, ${ }^{2}$ M. Waroquier ${ }^{2}$ and \\ P. Geerlings ${ }^{1}$ \\ 1 Eenheid Algemene Chemie (ALGC), Vrije Universiteit Brussel (VUB), Faculteit Wetenschappen, Pleinlaan 2, 1050 Brussels, Belgium \\ ${ }^{2}$ Laboratory of Theoretical Physics, Ghent University, Proeftuinstraat 86, B-9000 Ghent, Belgium \\ ${ }^{3}$ European Patent Office, Landsbergerstrasse 187, D-80687 Munich, Germany
}

Received 22 March 2004; Revised 16 June 2004; Accepted 16 June 2004

\begin{abstract}
The use of density functional methods for the elucidation of the structure of radiation-induced bio-radicals by comparison of computed and experimental EPR properties is discussed. Three case studies, radiation induced radicals of the amino acid alanine, steroid hormones and $\beta$-D-fructose, with increasing degree of uncertainty about the proposed radical structures, are investigated. Next to the analysis of the isotropic and anisotropic components of the hyperfine tensor, also the direction cosines of the principal axes of this tensor were investigated in greater detail in the case of the $\beta$-D-fructose radicals. Since all radicals considered in this contribution are formed in a solid matrix, also the question as to how to incorporate the effect of the molecular environment is addressed. It is concluded that the methodology outlined represents a powerful tool to aid experimentalists in the assignment of the contributions of various radicals contributing to the observed EPR spectra. Copyright $\odot 2004$ John Wiley \& Sons, Ltd.
\end{abstract}

KEYWORDS: EPR; density functional theory; bioradicals; radiation; structure; alanine; fructose

\section{INTRODUCTION}

\section{Prologue}

In this contribution, which is of a review type, electron paramagnetic resonance (EPR) properties are discussed as a source of information on the structure of radiation-induced bioradicals in the solid state. More precisely, we will show, via three case studies, how the comparison of the computed and experimental EPR properties of a series of radiationinduced radicals can be used to confirm or propose the structure of these radicals.

A large number of the studies on amino acids (in particular alanine) and sugars are motivated by their potential dosimetric applications. L- $\alpha$-Alanine, for example, is currently used as a reference dosimeter suitable over a wide dose range. ${ }^{1}$ In the first part, the hyperfine coupling constants for various candidate alanine radicals are discussed. ${ }^{2}$ For this system, many studies are available yielding a substantial amount of structural information and EPR spectroscopic properties of irradiated solid $\mathrm{L}-\alpha$-alanine. As a result, this system represents and ideal test case for the purpose of exploring and validating the DFT calculation of hfccs in

${ }^{\dagger}$ Dedicated to Professor M. Barfield on the occasion of his 70th birthday.

${ }^{*}$ Correspondence to: F. De Proft, Eenheid Algemene Chemie (ALGC), Vrije Universiteit Brussel (VUB), Faculteit Wetenschappen, Pleinlaan 2, 1050 Brussels, Belgium. E-mail: fdeprof@vub.ac.be radiation-induced bioradicals for the purpose of structure elucidation. At the end of this section, we will briefly mention the results of a study on the EPR properties of steroid radicals, ${ }^{3}$ where various experimental studies available agree on the identity of two different radicals whereas another two radicals form the subject of ongoing debate, to which the results of the theoretical analysis presented here can contribute significantly.

In the final section, a theoretical study is discussed on the tentative structures of fructose radicals, ${ }^{4}$ following an earlier study of the EPR spectra of irradiated sugars (e.g. glucose, fructose, sucrose) by Vanhaelewyn et al. ${ }^{5}$ The accurate calculation of hfccs in several candidate fructose radicals provides the experimentalist with valuable information on their possible structure but in turn requires a validated computational approach owing to its highly exploratory nature, as relatively few EPR studies are available. In addition to the computation of the isotropic and anisotropic components of the hyperfine interaction tensor, the eigenvectors of the proton hyperfine tensors also had to be investigated in order to elucidate the structure of the radicals involved.

In conclusion, the studies described in this work are characterized by a systematic increase in both the level of complexity of the molecular species considered and in the degree of uncertainty concerning the proposed radical structures. They represent case studies to which the essentials 
in the development of ESR studies can be attached and the resulting conclusions may be used as a general guide for future workers in the field dealing with structure elucidation of radiation-induced bioradicals using computed ESR properties.

It should be mentioned that during the last decade, a large number of studies have emerged on the theoretical prediction of EPR properties of biomolecular radicals, such as DNA, DNA bases and framework sugars, amino acids, steroids and sugars. These applications, focusing more on the performance of molecular orbital and density functional methods, have recently been compiled. ${ }^{6}$

\section{Electron paramagnetic resonance - basic principles}

Electron paramagnetic resonance (EPR) or electron spin resonance (ESR) is an important technique for studying the structures and properties of species with unpaired electrons. $^{7-9}$ Central in the field of EPR spectroscopy is the determination and interpretation of the hyperfine tensors describing the interaction between the unpaired electron(s) and the magnetic nuclei in the radical.

Complementary to the information available through a variety of experimental techniques, quantum chemical methods of ever increasing accuracy are available to gain insight into the particular EPR spectroscopic features of radicals. In this contribution, we review research work conducted in recent years dealing with the theoretical study of the EPR properties of bioradicals, often created by irradiation, and their importance in the radical identification process. The systems investigated range from amino acids and steroids to sugars, investigated both at the single molecule and radical in solid-state levels.

A simplified Hamiltonian can be proposed, adequate for treating most EPR and ENDOR spectra. For a system containing an unpaired electron and one interacting nucleus $N$, this spin Hamiltonian can be written as ${ }^{10}$

$$
H=\beta_{e} \mathbf{B}^{T} \mathbf{g S}-g_{N} \beta_{N} \mathbf{I}^{T} \mathbf{B}+\mathbf{S}^{T} \mathbf{A} \mathbf{I}
$$

The first term is the Zeeman interaction accounting for the interaction energy of the magnetic moment of the electron with the applied external magnetic field. It contains the Bohr magneton $\beta_{e}$, the magnetic field vector $\mathbf{B}$, the electron spin momentum operator $\mathbf{S}$ and the so-called $g$-tensor $\mathbf{g}$. As a rule, this electronic Zeeman interaction constitutes the dominant contribution to the EPR spectrum.

The Hamiltonian for the nuclear Zeeman interactions constitutes the second term of Eqn (1). In these terms, $\beta_{\mathrm{N}} g_{\mathrm{N}}$ is the nuclear magnetogyric factor, a constant for a given isotope, and I the nuclear spin operator. In general, this term only has a measurable contribution in ENDOR spectroscopy.

The final term of the spin Hamiltonian is the hyperfine interaction term, describing the interaction between the electronic and nuclear spin magnetic moments, where $\mathbf{A}$ is the hyperfine tensor. In general, this term is very important in ESR spectroscopy as the type and number of couplings (i.e. lines) in the ESR spectrum are determined by this interaction.
In organic $\pi$-type radicals, which will be central in this work, it appears that the $g$-tensor shows relatively little anisotropy and does not deviate substantially from the free electron value. For these kinds of systems, this parameter will not be very sensitive to (small) changes in geometry or conformation, even up to the point that the $g$-tensors of two different radicals of the types studied in this work can be virtually identical. As a consequence, we have not put our focus on the $g$-tensor.

As a result, the spin Hamiltonian can be further simplified to

$$
H=g \beta_{e} S_{z} B_{z}-g_{N} \beta_{N} I_{z} B_{z}+\mathbf{S}^{T} \mathbf{A I}
$$

We will now investigate the nature of this hyperfine interaction (giving rise to a contribution in the Hamiltonian denoted $H_{h f}$ ) and its direct importance for EPR spectroscopy. There are two types of magnetic interaction between the electron and the nuclear spins, i.e.

$$
H_{h f}=\mathbf{S}^{T} \mathbf{A I}=H^{(1)}+H^{(2)}
$$

The first part of the $H_{h f}$ Hamiltonian describes the direct dipolar interaction between the magnetic moments of the electronic and nuclear spins and has the following form:

$$
\begin{aligned}
H^{(1)}= & -\mu_{0} g \beta_{e} g_{N} \beta_{N}\left(\frac{1}{4 \pi \hbar^{2} r^{3}}\right) \\
& \times\left[\mathbf{S}^{T} \cdot \mathbf{I}-\frac{3\left(\mathbf{S}^{T} \cdot \mathbf{r}\right)\left(\mathbf{I}^{T} \cdot \mathbf{r}\right)}{r^{2}}\right]
\end{aligned}
$$

where $r$ is the electron-nucleus distance.

Once a suitable coordinate system is chosen that is fixed with respect to the sample (e.g. parallel to the crystal axes with the nucleus placed at the origin), the electron can be described by Cartesian coordinates $x, y$ and $z$. Averaging over the electron distribution transforms Eqn (4) to

$$
\begin{aligned}
H^{(1)}= & -\left(\frac{\mu_{0} g \beta_{e} g_{N} \beta_{N}}{4 \pi \hbar^{2}}\right) \times\left[\begin{array}{lll}
S_{x} & S_{y} & S_{z}
\end{array}\right] \\
& \times\left[\begin{array}{ccc}
\left\langle\frac{r^{2}-3 x^{2}}{r^{5}}\right\rangle & \left\langle\frac{-3 x y}{r^{5}}\right\rangle & \left\langle\frac{-3 x z}{r^{5}}\right\rangle \\
\left\langle\frac{-3 y x}{r^{5}}\right\rangle & \left\langle\frac{r^{2}-3 y^{2}}{r^{5}}\right\rangle & \left\langle\frac{-3 y z}{r^{5}}\right\rangle \\
\left\langle\frac{-3 z x}{r^{5}}\right\rangle & \left\langle\frac{-3 z y}{r^{5}}\right\rangle & \left\langle\frac{r^{2}-3 z^{2}}{r^{5}}\right\rangle
\end{array}\right] \\
& \times\left[\begin{array}{lll}
I_{x} & I_{y} & I_{z}
\end{array}\right]
\end{aligned}
$$

or

$$
H^{(1)}=-\left(\frac{\mu_{0} g \beta_{e} g_{N} \beta_{N}}{4 \pi \hbar^{2}}\right) \mathbf{S}^{T} \mathbf{T I}
$$

It can be easily derived from this equation that an electron in an s-orbital has no net magnetic interaction with the nucleus, since the electron distribution in such an orbital is spherically symmetrical. Consequently, this interaction cannot account for the hyperfine coupling that is observed in the hydrogen atom.

The second part of the $H_{h f}$ Hamiltonian [Eqn (3)] therefore stands for a second hyperfine interaction mechanism, the so-called Fermi-contact interaction:

$$
H^{(2)} \approx \frac{2}{3} g \beta_{e} g_{N} \beta_{N} \mu_{0} \delta(\mathbf{r}) \mathbf{S}^{T} \mathbf{I}
$$


In finite perturbation theory, the expectation value of $H^{(2)}$ with respect to the total wavefunction is needed. For practical reasons, only the integration in coordinate space is performed, resulting in an expression with only spin operators:

$$
H^{(2)}=\frac{2}{3} g \beta_{e} g_{N} \beta_{N} \mu_{0}|\Psi(\mathbf{r}=0)|^{2} \mathbf{S}^{T} \cdot \mathbf{I}=A_{0} \mathbf{S}^{T} \cdot \mathbf{I}
$$

where $\Psi(\mathbf{r}=0)$ is the single-particle wavefunction of the unpaired electron at the position of the nucleus. The factor

$$
A_{0}=\frac{2}{3} g \beta_{e} g_{N} \beta_{N} \mu_{0}|\Psi(\mathbf{r}=0)|^{2}
$$

in this expression is called the isotropic hyperfine coupling constant.

Taking into account expressions (6) and (9), the hyperfine matrix $\mathbf{A}$ of Eqn (2) can be divided in an isotropic and an anisotropic part:

$$
\mathbf{A}=A_{0} \mathbf{1}_{3}+\mathbf{T}
$$

where $\mathbf{1}_{\mathbf{3}}$ is a $3 \times 3$ unit matrix.

Free radicals are often encountered as structural species in a rigid matrix (e.g. a crystal lattice), a situation with which we will be almost exclusively dealing in this contribution. As a result, a maximum amount of information can be obtained if the EPR spectra are obtained as a function of the orientation of the crystal in the magnetic field. The analysis of the data resulting from such an approach requires, however, a detailed understanding of the nature of anisotropic hyperfine interactions. It is the interaction between the electron and a nuclear dipole at some distance away [see Eqn (4)] that will give rise to the observed anisotropy in the hyperfine interaction and this will now be discussed in detail.

It can be shown that the hyperfine matrix $\mathbf{A}$ itself is not available experimentally. However the symmetrical hyperfine matrix $\mathbf{A} \mathbf{A}^{\mathrm{T}}$, to within a factor \pm 1 , can be obtained from a set of measurements at different orientations of the magnetic field.

The procedure for evaluating the elements of the hyperfine matrix is the following. The magnitude of the symmetrical hyperfine matrix is

$$
A^{2}=\mathbf{n}^{\mathrm{T}} \mathbf{A A n}
$$

where $\mathbf{n}$ is a unit vector in the direction of $\mathrm{B}$, so that

$$
\mathbf{n}=\frac{\mathbf{B}}{B}
$$

Taking into account the form of this unit vector expressed in spherical polar coordinates:

$$
\mathbf{n}^{T}=\left[\begin{array}{lll}
c_{x} & c_{y} & c_{z}
\end{array}\right]=\left[\begin{array}{lll}
\sin \theta \cos \phi & \sin \theta \sin \phi & \cos \theta
\end{array}\right]
$$

$\mathbf{A}^{2}$ can be written as

$$
\begin{aligned}
A^{2}= & {\left[\begin{array}{ccc}
c_{x} & c_{y} & c_{z}
\end{array}\right] } \\
& \times\left[\begin{array}{lll}
\left(\mathbf{A A}^{T}\right)_{x x} & \left(\mathbf{A A}^{T}\right)_{x y} & \left(\mathbf{A A}^{T}\right)_{x z} \\
& \left(\mathbf{A A}^{T}\right)_{y y} & \left(\mathbf{A A}^{T}\right)_{y z} \\
& & \left(\mathbf{A A}^{T}\right)_{z z}
\end{array}\right]\left[\begin{array}{l}
c_{x} \\
c_{y} \\
c_{z}
\end{array}\right]
\end{aligned}
$$

or

$$
\begin{aligned}
= & \left(\mathbf{A A}^{T}\right)_{x x} \sin ^{2} \theta \cos ^{2} \phi+2\left(\mathbf{A} \mathbf{A}^{T}\right)_{x y} \sin ^{2} \theta \cos \phi \sin \phi \\
& +\left(\mathbf{A} \mathbf{A}^{T}\right)_{y y} \sin ^{2} \theta \sin ^{2} \phi+2\left(\mathbf{A} \mathbf{A}^{T}\right)_{x z} \cos \theta \sin \theta \cos \phi \\
& +2\left(\mathbf{A A}^{T}\right)_{y z} \cos \theta \sin \theta \sin \phi+\left(\mathbf{A} \mathbf{A}^{T}\right)_{z z} \cos ^{2} \theta
\end{aligned}
$$

Consequently, one needs to determine the six independent components of the symmetric $\mathbf{A A}^{\mathrm{T}}$ tensor, which can, in principle, be accomplished by six different measurements at suitable crystal orientations. Once this matrix has been determined, it can be diagonalized, yielding the principal axes and principal values. Next, taking the square root of its three principal values yields the magnitudes of the principal values of the symmetrized hyperfine matrix $\left(\mathbf{A}+\mathbf{A}^{\mathrm{T}}\right) / 2$.

Finally, the matrix is written in the form of Eqn (10); the isotropic hyperfine coupling constant $A_{0}$, together with the elements of the traceless tensor $\mathbf{T}$ and their respective eigenvectors (defining the principal axes) are the values most frequently reported in experimental studies.

\section{Computational considerations}

$A b$ initio computations of hyperfine structures have been performed for about 30 years. ${ }^{11-14}$ Only when calculations could be performed that included electron correlation and appropriately well-chosen basis sets could substantial increase in accuracy be obtained. ${ }^{15-24}$ Many studies on the performance of various theoretical approaches in the computation of hyperfine structures of molecules have been performed. One of the main conclusions drawn is that isotropic hyperfine coupling constants (hfccs) are extremely sensitive to the geometry of the system, necessitating careful geometry optimizations. ${ }^{15-24}$

In the literature, a number of studies have emerged concentrating on the performance of DFT computational methods in the computation of hfcc's for small molecules. ${ }^{6,25-45}$

A general study of the performance of different theoretical methods in the prediction of radical hfccs was performed by Gauld et al. ${ }^{42}$ They investigated a series of small radicals, more specifically $\mathrm{OH}, \mathrm{H}_{2} \mathrm{O}^{+}, \mathrm{CN}, \mathrm{HCN}^{-}, \mathrm{FCN}^{-}, \mathrm{HCCH}^{-}$, $\mathrm{CH}_{3}, \mathrm{CH}_{4}{ }^{+}, \mathrm{NH}_{2}, \mathrm{NO}_{2}$ and $\mathrm{H}_{2} \mathrm{CO}^{+}$, and applied both ab initio-post-Hartree-Fock (HF) and DFT methods in combination with a large number of extended basis sets. In this work, the QCISD (quadratic configuration interaction with single and double excitations) method $^{46}$ was found to yield results that were superior to both other postHF methods and DFT. For the DFT approaches, the best overall accuracy was found for the B3LYP functional ${ }^{47,48}$ in combination with either the Pople $6-311 G+(2 d f, p)^{49}$ or the IGLO-III basis set. ${ }^{50}$ However, with respect to the issue of the basis set, it was suggested that for larger anions and neutral radicals, the $6-311 \mathrm{G}(\mathrm{d}, \mathrm{p})$ set would represent a reasonable compromise between accuracy and computational cost. Adamo et al..$^{33}$ analysed the performance of different hybrid density functionals in the computation of structural and electronic characteristics of organic $\pi$ radicals. They performed extensive DFT calculations of geometric and thermochemical properties, infrared frequencies and hyperfine coupling constants for a series of representative carboncentered $\pi$ radicals. It was concluded that, overall, B3LYP 
geometries and hfccs for both $\mathrm{H}$ and $\mathrm{C}$ atoms show approximately the same accuracy as the most sophisticated post-HF methods.

Another assessment was put forward by Batra et al. ${ }^{40}$ They applied semiempirical, HF and DFT methods in the calculation of hfccs of a set of 17 organic neutral and charged radicals, chosen to cover a broad range of compounds. It was concluded that the combination of UHF $/ 3-21 G^{*}$ geometries with single-point DFT calculations of the EPR properties at the BLYP /6-31G* or B3LYP /6-31G* level yields maximum deviations between computed and experimental hfccs of about $10 \%$. The performance of other, more recently proposed density functionals has also been investigated and has recently been reviewed. ${ }^{6}$ In that review, attention was also been focused on other effects that determine the magnetic properties of organic free radicals, such as the effect of the environment and the effect of the vibrational averaging of the properties. These two matters will also be encountered in the present review.

From all these studies, it can be generally concluded that DFT methods provide a good quality / cost ratio for the study of hyperfine interactions are hence are the methods of choice to use with large molecules.

Within a Kohn-Sham density-functional scheme, the hyperfine properties can be obtained once the unpaired ground state spin density is known: $:^{6,34,37,51}$

$$
\rho_{s}=\sum_{\mu} \sum_{\nu} P_{\mu \nu}^{\alpha-\beta} \phi_{\mu} \phi_{v}
$$

It should be mentioned that the atomic spin densities reported in this work are always obtained within the Mulliken formalism. We have

$$
A_{i s o}=\frac{2}{3} g \beta_{e} g_{N} \beta_{N} \sum_{\mu} \sum_{\nu} P_{\mu \nu}^{\alpha-\beta}\left\langle\phi_{\mu}(\mathbf{r})|\delta(\mathbf{r})| \phi_{\nu}(\mathbf{r})\right\rangle
$$

and

$$
\begin{aligned}
T_{k l}= & -\frac{g \beta_{e} g_{N} \beta_{N} \mu_{0}}{4 \pi \hbar^{2}} \sum_{\mu} \sum_{\nu} P_{\mu \nu}^{\alpha-\beta} \\
& \left\langle\phi_{\mu}(\mathbf{r})\left|\frac{\left(\mathbf{r}^{2} \delta_{k l}-3 r_{k} r_{l}\right)}{r^{5}}\right| \phi_{\nu}(\mathbf{r})\right\rangle
\end{aligned}
$$

for the $k, l$ component of the anisotropic hyperfine interaction tensor $\mathbf{T}$.

\section{Computational approach}

The radical species that will be treated in this work are all relatively large, organic (mostly $\pi$-type) radicals for which hfccs of mainly protons are to be calculated. In this case, the available literature data univocally suggest that the combination of a hybrid functional with either a moderate-size Pople basis set or a purposely built basis set can provide a very cost-effective computational method. It is widely accepted that the B3LYP/6-31G(d) level yields geometries of sufficient quality for a broad range of chemical applications. $^{51}$

Next, a comparative study was undertaken of the performance of a variety of computational levels towards hfcc calculations in a selection of radicals that will be presented in the following sections. The results displayed in Fig. 1 show that indeed only DFT calculations offer a satisfactory agreement with experimental values and this at a computational cost that is of the same order of magnitude as the HF approaches. Additionally, the combination of the B3LYP functional with the $6-311 G(\mathrm{~d})$ basis set results in the best overall quality (mean deviation from experimental values $<5 \%$ ).

As a result, we chose the triple zeta Pople basis set $6-311 G(d)$ or $6-311 G(d, p)$, taking into account the results of available studies and the nature of the molecular species under study. In some selected cases, calculations with the

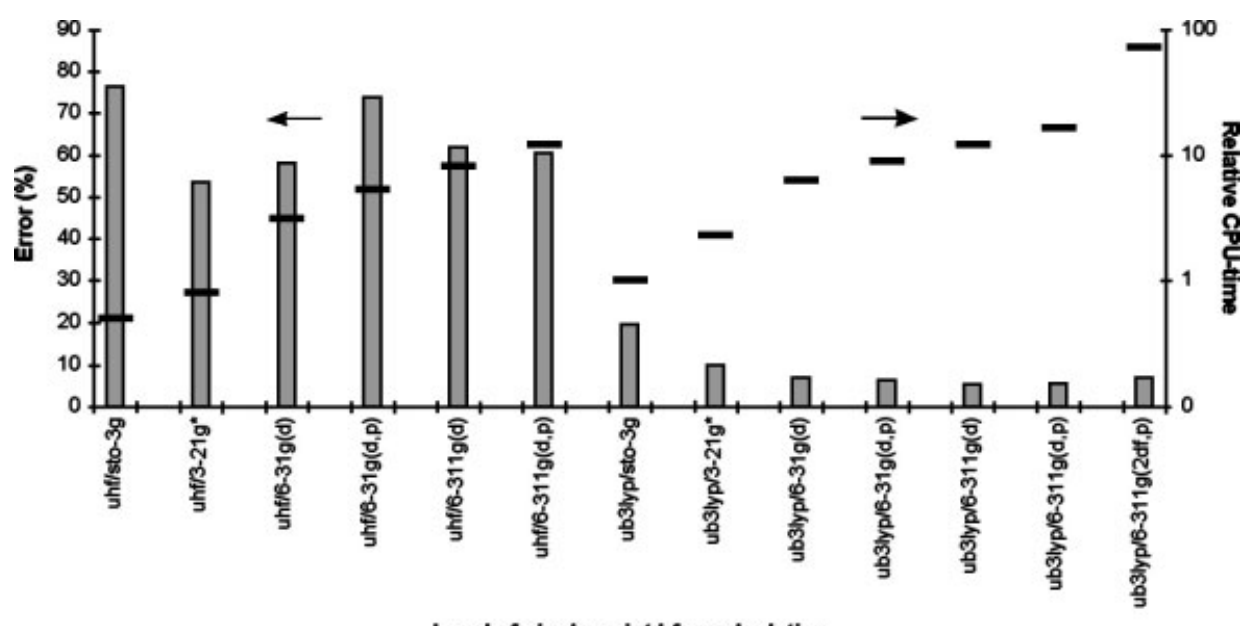

Level of single-point hfcc calculation

Figure 1. Evolution of mean errors (bars) and mean relative CPU times (lines) as a function of the single-point hfcc calculation level for selected alanine and steroid radicals studied in this work. Protons whose isotropic hfcc value is included in the calculations: $\mathrm{H} 7-\mathrm{H} 10$ (alanine radical R1), $\mathrm{H} 8-\mathrm{H} 13$ (alanine radical R2), H8-H11 (alanine radical R3), H4, H6, H7A and H7B (steroid radical R1), $\mathrm{H} 1 \mathrm{~A}, \mathrm{H} 1 \mathrm{~B}, \mathrm{H} 2$ (steroid radical R2). The geometries were optimized at the B3LYP/6-31G(d) level; errors are defined as the percentage deviation of the respective reference experimental values. All mean CPU times are relative to the CPU time required for the UB3LYP/STO-3G single-point calculation. 
more involved IGLO-III ${ }^{50}$ or EPR-III ${ }^{6,34}$ basis sets, specifically designed for the computation of NMR and EPR properties, were performed.

Finally, we want to stress that all radicals studied here are embedded in the solid state. Hence another item emerges, namely how to treat the molecular environment. In the simplest case one can adopt a single-molecule approach. In this approximation, it can be expected that from the geometry optimization of the single radical, one will not necessarily obtain the geometry corresponding to the observed EPR properties. Basically two approaches, both described in this work, can then be adopted to determine the valid conformation of the radical. In a first strategy, one can vary the geometric parameters of the isolated radical in order to obtain maximum agreement with the experimental findings. In a second, more refined approach, one can explicitly take into account the radical neighbourhood, by performing either cluster or periodic calculations.

\section{ALANINE-DERIVED RADICALS: A TEXTBOOK CASE: STRENGTHS, WEAKNESSES, OPPORTUNITIES AND THREADS}

\section{Introduction}

In this section, we will focus on a first application of DFT calculations to predict the EPR properties of bioradicals. The amino acid $\mathrm{L}-\alpha$-alanine in its solid state is an ideal candidate for a wide range of applications in EPR dosimetry, because it yields good dose factors, a linear signal response over a wide dose range, excellent fading characteristics and limited dependence on dose rate, radiation quality and environmental factors (e.g. temperature and humidity). ${ }^{1}$ As a result, many EPR and ENDOR spectroscopic studies on this species have emerged and many experimental data on electronic $g$-factors and hyperfine coupling constants of the magnetic nuclei in this radical are available. The radiationinduced radicals of this species are therefore ideal candidates for theoretical calculations.

A simplified picture of the different radiation-induced alanine radicals occurring at different temperatures, studied in this section, is given in Fig. 2. In the crystal lattice, alanine occurs in the zwitterionic form. As shown in Fig. 2, irradiation of the crystal at $77 \mathrm{~K}$ yields a primary alanine radical anion. ${ }^{52}$ When the crystal is gradually warmed, this anion degrades by deamination, turns into an unstable radical conformation at $150 \mathrm{~K}^{53}$ and finally into a stable radical conformation at about $220 \mathrm{~K}^{54,55}$ Both the stable and the unstable radicals have the same chemical structure, $\mathrm{CH}_{3} \mathrm{C}^{\bullet} \mathrm{HCOO}^{-}$, as represented in Fig. 2, but differ with respect to their relative orientation in the crystal lattice and internal bond lengths and angles. According to the model of Itoh and Miyagawa, ${ }^{56}$ the EPR absorption is to be interpreted as a statistical average of the two types of radicals. While it has been commonly assumed that the room temperature EPR spectrum of polycrystalline alanine could be ascribed to the radical $R 1,^{57-63}$ there have been speculations on the possible coexistence of several stable radical species, possibly originating from primary radicals other than that shown in Fig. 2. ${ }^{64}$ Recently however, Sagstuen and co-workers

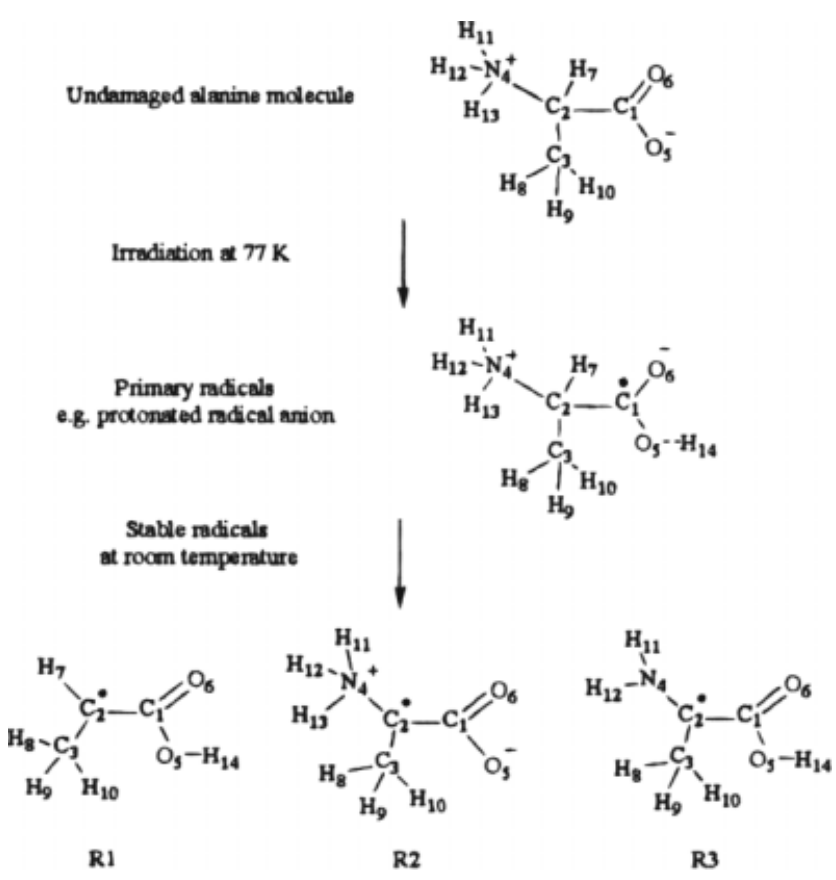

Figure 2. $L-\alpha$-Alanine and derived radicals studied in this section. Reproduced with permission from Lahorte $\mathrm{P}$ et al. (Ref. 2). Copyright 1999 American Chemical Society.

presented experimental evidence for the existence of R2 in irradiated alanine. ${ }^{64-66}$ In these contributions, evidence for the existence of a third radical R3 was put forward. ${ }^{64-66}$

In the next section, the EPR properties of the abovementioned alanine radicals are compiled and discussed at the single-molecule level. For radical R2, which is the simplest adduct of the undamaged alanine molecule, comparison is made with calculations including the molecular environment.

\section{The primary radical}

The constrained optimization of this species mimicking its geometry in the crystal lattice did not result in good agreement of the EPR properties with experiment. Therefore, a full geometry optimization of this species was performed, followed by a series of single-point calculations of the hyperfine coupling constants upon rotation of the carboxyl group over $360^{\circ}$ around the $\mathrm{C} 1-\mathrm{C} 2$ bond axis, with a stepsize of $18^{\circ}$. Next, for a series of fixed values of this angle (between 0 and $180^{\circ}$ ), a partial geometry optimization was performed. The results of these calculations are given in Fig. 3.

The large isotropic hyperfine coupling constant for the carbon atom $\mathrm{C} 1(251.4 \mathrm{MHz})$ indicates that the $\mathrm{CO}_{2}$ group deviates from planarity at the radical centre on $\mathrm{C} 1$. Experimentally, a deviation of $8^{\circ}$ was suggested, which is in good agreement with the $14^{\circ}$ resulting from the DFT geometry optimization. As can be seen from Fig. 3, only the primary radical conformation with a rotation angle of $180^{\circ}$ yields a good identification with the experimental hfcc. The corresponding hfccs are displayed in Table 1 . The agreement with the experimental values is very good (difference $<5 \%$ ). At a qualitative level, Fig. 3 also displays the typical $\beta$ proton 
Table 1. Calculated versus experimental hyperfine coupling constants for the various alanine-derived radicals. Reproduced with permission from Lahorte P et al. (Ref. 2). Copyright 1999 American Chemical Society

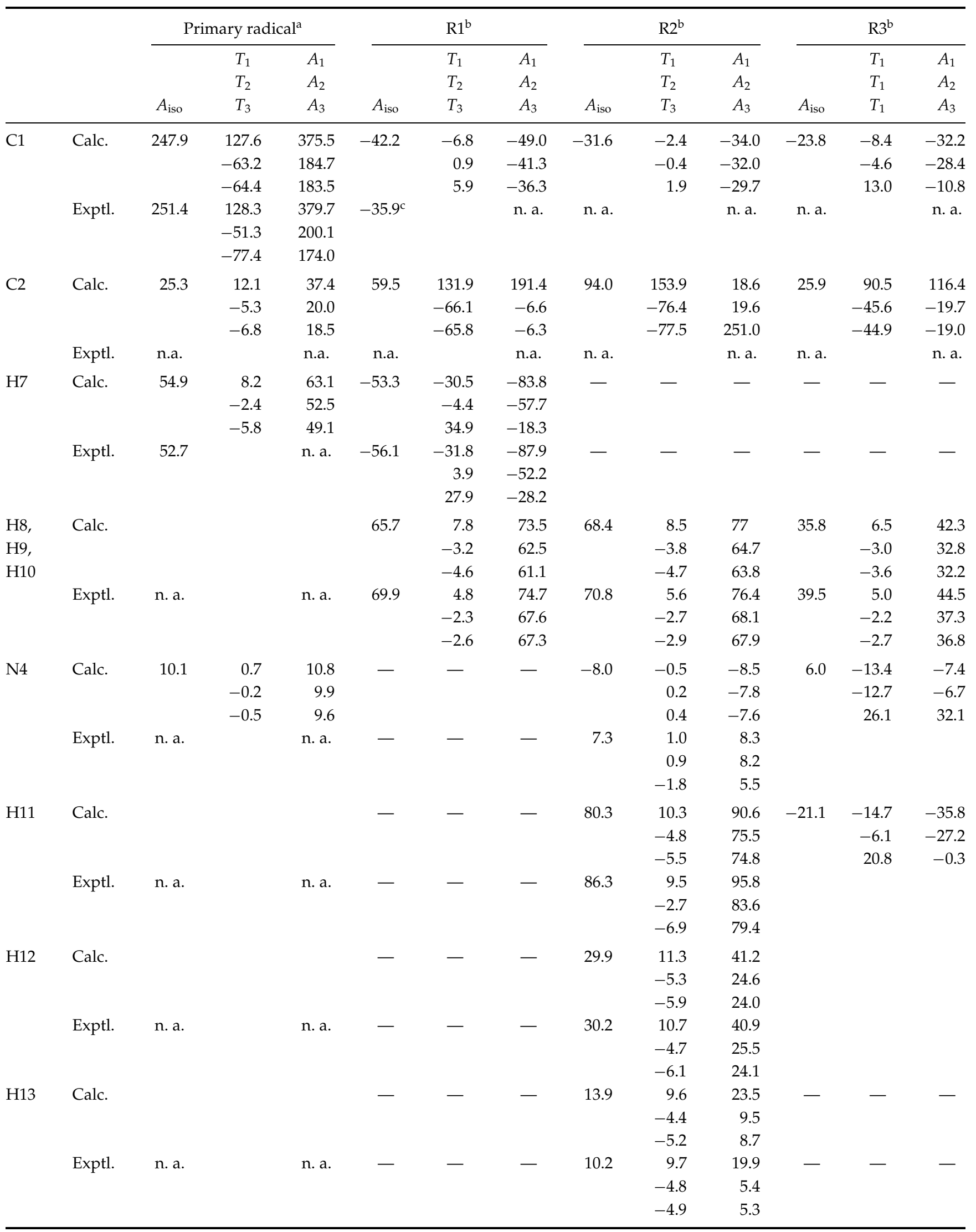

${ }^{a}$ Ref. 62

${ }^{\mathrm{b}}$ Ref. 63.

${ }^{\mathrm{c}}$ Experimentally, the absolute value is reported. 


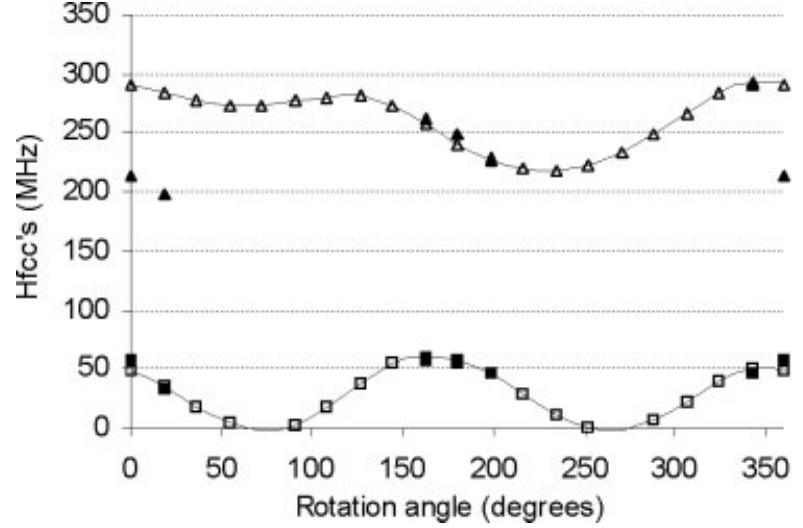

Figure 3. Angular variation of the $\mathrm{C} 1(\triangle)$ and $\mathrm{H} 7(\square)$ hfccs upon rotation of the carboxyl group around the $\mathrm{C} 1-\mathrm{C} 2$ bond axis in the primary alanine radical. Filled symbols $[(\boldsymbol{\Delta})$ and $(\boldsymbol{\square})]$ are used to denote the respective values when performing reoptimization of all internal coordinates upon rotation. UB3LYP/6-311G(d)//B3LYP/6-31G(d) level of calculation. All values are in $\mathrm{MHz}$. Reproduced with permission from Lahorte $\mathrm{P}$ et al. (Ref. 2). Copyright 1999 American Chemical Society.

character of atom $\mathrm{H} 7$, as the magnitude of the coupling varies with the square of the cosine of the rotation angle.

\section{The stable alanine radical $\mathrm{R} 1$}

The B3LYP/6-31G(d) geometry of this radical is depicted in Fig. 4.

The EPR properties of this species are also listed in Table 1. In their experiment with $\left[1-{ }^{13} \mathrm{C}\right]-\mathrm{L}-$ alanine, Sinclair and Hanna ${ }^{61,62}$ reported an essentially isotropic splitting with an absolute value of $35.9 \mathrm{MHz}$ for the $\mathrm{C} 1$ nucleus of the carboxyl group. As can be seen, the agreement with experiment is very good for this nucleus. Moreover, the calculations confirm that the unpaired electron is primarily located at the carbon $\mathrm{C} 2$, as indicated by the (Mulliken) spin populations for this atom being 0.88 and 0.89 at the B3LYP/6-311G(d) and B3LYP/IGLO III levels, respectively. As a result, the hyperfine couplings in this radical will be mainly due to the interaction with the $\alpha$-proton $\mathrm{H7}$

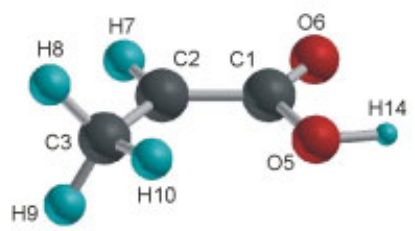

R1

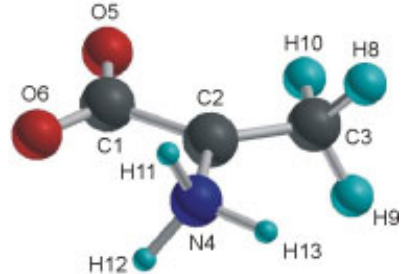

R2

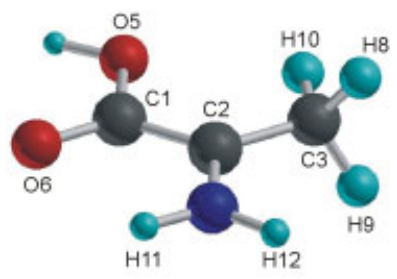

$\mathbf{R 3}$

Figure 4. Molecular structures of the radicals R1, R2 and R3. and the three symmetry equivalent, rotationally averaged methyl $\beta$-protons. The $\alpha$-proton interaction in the stable alanine radical R1 has been extensively investigated. As can be seen from Table 1, both isotropic and anisotropic calculated $\alpha$-proton hyperfine coupling constants are in very good agreement with the experimental values. It should be remarked, however, that different experimental techniques used to determine these coupling constants can give rise to different numerical values. ${ }^{2}$

In a next step, the dependence of the hfccs of the $\beta$-methyl protons on the rotation of the methyl group over an angle of $180^{\circ}$ in steps of $18^{\circ}$ about the C2-C3 axis was investigated. The properties obtained using this procedure are plotted in Fig. 5 , which clearly reveals the $\beta$-proton character of the methyl protons.

It has been shown in several radical systems that isotropic interactions of $\beta$-protons may vary considerably between approximately 10 and $140 \mathrm{MHz}$, with symmetry equivalent (i.e. rotationally averaged) protons typically showing splittings of about $70 \mathrm{MHz}$. In general, anisotropic interactions in $\beta$-protons are limited. In the B3LYP/6-31G(d) optimized geometry, the radical has $C_{s}$ symmetry, showing a perfectly planar radical centre, the two methyl protons $\mathrm{H} 8$ and $\mathrm{H} 9$ being symmetrically above and below the symmetry plane. The dihedral angle $\theta$ of the $\mathrm{H} 8-\mathrm{C} 3$ and $\mathrm{H} 9-\mathrm{C} 3$ bonds with the $\mathrm{H} 10-\mathrm{C} 3$ bond is 121.4 and $-121.4^{\circ}$, respectively. Hence the proton $\mathrm{H} 8$ would make a dihedral angle of $31.4^{\circ}$ with the lone electron orbital (LEO) in a $\pi$-type radical. As a result, rotation of the methyl group over this angle results in a maximum contribution of the $\mathrm{H} 8$ to the singly occupied molecular orbital (SOMO), yielding a maximum hyperfine coupling constant, which can be clearly seen in Fig. 5(a).
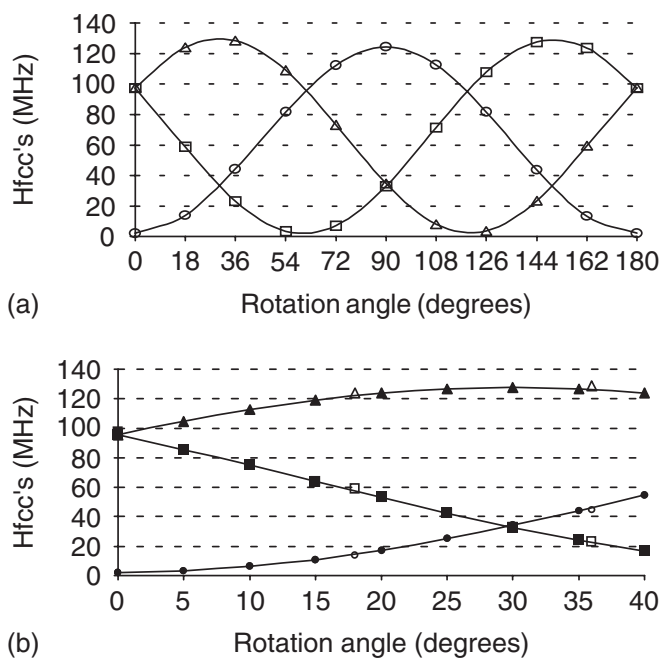

Figure 5. Angular variation of the methyl proton hfcc's on rotation of the methyl group around the $\mathrm{C} 2-\mathrm{C} 3$ bond axis in the radical R1. UB3LYP/6-311G(d)//B3LYP/6-31G(d) level of calculation. All values are in $\mathrm{MHz}$. (A) Using original optimized internal coordinates: $(\triangle) \mathrm{H}$; $(\square) \mathrm{H}$; ( (O) H10. (B) Effect of reoptimization of methyl group internal coordinates upon

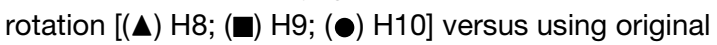
optimized internal coordinates $[(\triangle) H 8$; $(\square) H$; ; (O) H10]. Reproduced with permission from Lahorte $\mathrm{P}$ et al. (Ref. 2). Copyright 1999 American Chemical Society. 
The slightly smaller interaction of H10 is completely due to the use of optimized internal coordinates in the single-point calculations. Whereas for the $\mathrm{H} 8$ and $\mathrm{H} 9$ protons the $\mathrm{H}-\mathrm{C} 3$ bond lengths are both $1.100 \AA$ and the $\mathrm{H}-\mathrm{C} 3-\mathrm{C} 2$ bond angles $110.8^{\circ}$, the $\mathrm{H} 10-\mathrm{C} 3$ bond length and $\mathrm{H} 10-\mathrm{C} 3-\mathrm{C} 2$ angle are $1.091 \AA$ and $111.9^{\circ}$, respectively. This results in a maximum isotropic hfcc of $124 \mathrm{MHz}$ for $\mathrm{H} 10$ and $129 \mathrm{MHz}$ for $\mathrm{H} 8$ and $\mathrm{H} 9$, a relative difference of about $4 \%$. In order to eliminate this effect, a reoptimization of the geometry of the methyl group was performed along each step of the rotation. The methyl group was rotated around the $\mathrm{C} 2-\mathrm{C} 3$ axis over $40^{\circ}$, with a step size of $5^{\circ}$, containing the regions where the best agreement with the experimental hfcc's occurs. The results are displayed in Fig. 5(b). As can be seen, allowing for a partial reoptimization of the internal coordinates has only a very small effect (on average less than $3 \%$ ) on the hfccs of the methyl protons.

It can be invoked from the data in Table 2 that the calculated average value of $65.7 \mathrm{MHz}$ for the isotropic hfcc of the methyl protons deviates less than $10 \%$ from the listed experimental value. This is also the case when a comparison is made with other experimental values. ${ }^{2}$

The values reported by Matsuki and Miyagawa ${ }^{55}$ refer to the above-mentioned unstable conformation of the radical R1 in the crystal lattice. In this unstable conformation, the methyl group motion is free at $77 \mathrm{~K}$. In contrast, the methyl group motion is frozen at this temperature for the stable conformation, resulting in individualized hyperfine tensors for the three methyl protons. These were reported by Miyagawa and Itoh $^{54}$ and the principal values are given in Table 2.

The calculated results reported in Table 2 are the best fits from Fig. 5(a), which were obtained by rotating the methyl group over $13^{\circ}$ towards the LEO. This results in a final angle of $18.4^{\circ}$ for proton $\mathrm{H} 8$, which is in very good agreement with the experimental value of $17^{\circ}$. Other possibilities that arise from Fig. 5(a) simply arise from symmetry equivalence of the three methyl protons. It can therefore be concluded that, solely by considering the hfccs, the conclusions drawn from experiment can be confirmed, i.e. that in the crystal

Table 2. Calculated and experimental methyl proton hfccs in the stable radical R1 at $77 \mathrm{~K}$ : UB3LYP/6-311G(d)//B3LYP/6$31 \mathrm{G}(\mathrm{d})$ level of calculation; all values are in $\mathrm{MHz}$. Reproduced with permission from Lahorte P et al. (Ref. 2). Copyright 1999 American Chemical Society

\begin{tabular}{|c|c|c|c|c|c|c|}
\hline \multicolumn{2}{|c|}{$\mathrm{H} 8$} & \multicolumn{2}{|c|}{ H9 } & \multicolumn{2}{|c|}{ H10 } & \multirow[b]{2}{*}{$\begin{array}{c}\text { Reference, } \\
\text { (method, } \\
\text { temperature) }\end{array}$} \\
\hline$A_{\text {iso }}$ & $\begin{array}{l}A_{1} \\
A_{2} \\
A_{3}\end{array}$ & $A_{\text {iso }}$ & $\begin{array}{l}A_{1} \\
A_{2} \\
A_{3}\end{array}$ & $A_{\text {iso }}$ & $\begin{array}{l}A_{1} \\
A_{2} \\
A_{3}\end{array}$ & \\
\hline 121.3 & $\begin{array}{l}129.2 \\
118.0 \\
116.6\end{array}$ & 77.6 & $\begin{array}{l}84.1 \\
75.1 \\
73.7\end{array}$ & 14.9 & $\begin{array}{l}19.3 \\
15.1 \\
10.1\end{array}$ & $54(\mathrm{EPR}, 77 \mathrm{~K})$ \\
\hline 118.5 & $\begin{array}{l}126.5 \\
115.4 \\
113.6\end{array}$ & 71.3 & $\begin{array}{l}79.0 \\
68.0 \\
66.8\end{array}$ & 10.8 & $\begin{array}{r}18.2 \\
7.3 \\
6.9\end{array}$ & 2 \\
\hline
\end{tabular}

lattice, the 'frozen' methyl group takes up a non-symmetrical conformation with respect to the plane of the radical.

\section{Radical R2}

Figure 4 shows the B3LYP/6-31G(d) geometry of the radical $\mathrm{R} 2$, which is formed by abstraction of the $\alpha$-proton in alanine. At the UB3LYP /6-311G(d) level, the total spin density on the central carbon atom C2 amounts to 0.93 (0.96 with the IGLO III basis set), which is substantially higher than the previously estimated value of 0.72 reported by Sagstuen et al. ${ }^{64}$ It can therefore be expected that the hyperfine interaction will be mainly due to the methyl and amino protons. For the methyl protons, our calculations suggest comparable couplings to those obtained for radical R1, as can be seen from Table 1 .

After a complete optimization of the radical (constrained for the zwitterionic form), the amino group was rotated over $180^{\circ}$ around the $\mathrm{N} 4-\mathrm{C} 2$ bond axis in steps of $18^{\circ}$; at each of the resulting conformations, single-point computations of the hfccs were performed, assuming a dihedral angle of $120^{\circ}$ between the respective $\mathrm{N} 4-\mathrm{H}$ bonds. The results are presented in Fig. 6.
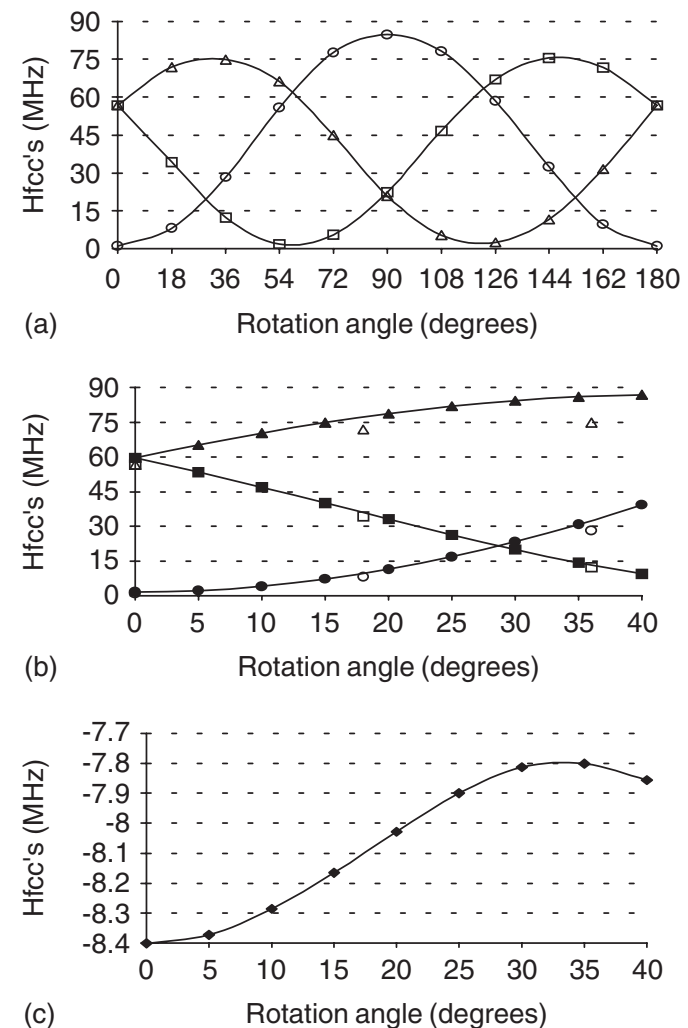

Figure 6. Angular variation of the amino proton $(a, b)$ and nitrogen N4 (c) hfccs on rotation of the amino group around the $\mathrm{N} 4-\mathrm{C} 2$ bond axis in the radical $\mathrm{R} 2$.

UB3LYP/6-311G(d)//B3LYP/6-31G(d) level of calculation; all values are in $\mathrm{MHz}$. (A) Using original optimized internal coordinates: $(\triangle) \mathrm{H} 11$; $(\square) \mathrm{H} 12$; $(\bullet) \mathrm{H} 13$; (B) effect of reoptimization of amino group internal coordinates upon rotation $[(\boldsymbol{\Delta}) \mathrm{H} 11 ;(\boldsymbol{\square}) \mathrm{H} 12 ;(\bullet) \mathrm{H} 13]$ versus using original optimized internal coordinates $[(\triangle) \mathrm{H} 11 ;(\square) \mathrm{H} 12$; $(\bullet) \mathrm{H} 13]$. Reproduced with permission from Lahorte P et al. (Ref. 2). Copyright 1999 American Chemical Society. 
Analogous to the methyl protons, the amino protons show a typical $\beta$-proton character, but with a smaller average isotropic coupling of $41.4 \mathrm{MHz}$, which is in good agreement with the experimental result of $42.2 \mathrm{MHz}$. Again, the fact that the angular variation of the hfccs for the three protons $\mathrm{H} 11, \mathrm{H} 12$ and H13 is not entirely the same is due to the use of the optimized geometry during the variation of this angle. In order to eliminate this effect, the amino group was reoptimized during each rotation step; more specifically, the amino group was rotated over $40^{\circ}$ around the N4-C2 axis in steps of $5^{\circ}$. The amino proton bond lengths and bond angles, together with the $\mathrm{N} 4-\mathrm{C} 2$ bond length, were allowed to relax while all other geometry parameters were fixed. As can be seen from Fig. 6, contrary to the situation for radical R1, there is a substantial effect $\left(\sim 10 \%\right.$ at a rotation angle of $\left.35^{\circ}\right)$ associated with this procedure for the hfcc of proton H11. As a result, the remainder of the discussion for this radical will be conducted using the data resulting from the latter procedure.

Experimentally, the free rotation of the amino group around the $\mathrm{N} 4-\mathrm{C} 2$ axis is prevented by the nearest neighbour atoms in the crystal lattice, which results in three individual hyperfine coupling tensors for the amino protons. The best agreement with the experimental values is obtained for a rotation angle of $22.4^{\circ}$, which results in a dihedral angle of the $\mathrm{H} 11-\mathrm{N} 4$ bond with the LEO of $7.6^{\circ}$. The corresponding hfcc values are given in Table 1. It can therefore be concluded that the amino group is slightly 'skewed' from the radical plane in the radical R2 conformation. This is in line with the conclusions of Sagstuen et al. ${ }^{64}$ who suggested a value of $7.3^{\circ}$, assuming a perfectly planar radical backbone. In their work, 8.3, 8.2 and $5.5 \mathrm{MHz}$ were proposed for the principal values of the N4 hyperfine interaction. This proposal was obtained by downscaling the experimental glycine values, reported by Deigen et al. by a factor of $0.75 / 0.90$ of the C2 carbon spin population. ${ }^{67}$ The variation of the $\mathrm{N} 4$ isotropic hfcc upon rotation of the amino group is also shown in Fig. 6, confirming both the sign and the magnitude of the N4 atom hfcc.

\section{Radical R3}

The geometry of this radical is depicted in Fig. 4; at the B3LYP/6-311G(d) level, the spin population amounts to 0.24 on the nitrogen $\mathrm{N} 4$ atom and to 0.53 and 0.16 for the C2 and O6 atoms, respectively, the corresponding values obtained with the IGLO III basis set being virtually identical. The average value for the isotropic hfcc of the methyl protons amounts to $37.1 \mathrm{MHz}$ (IGLO III level), whereas the anisotropic values are 43.5, 34.2 and $33.5 \mathrm{MHz}$, respectively. These values are comparable to the $6-311 \mathrm{G}(\mathrm{d})$ values, listed in Table 1. Sagstuen et al. assigned two hyperfine coupling tensors for the methyl protons corresponding to two conformations of the radical R3 in the crystal lattice. ${ }^{64}$ Apart from the H8, H9, H10 values present in Table 1, they also reported a value of $33.1 \mathrm{MHz}$ for the isotropic hfcc and $37.7,30.9$ and 30.8 as the principal values for a second radical R3 conformation in the crystal lattice. Again, the agreement between the theoretical and experimental values is excellent. However, the current study does not allow for investigation of the influence of the two above-mentioned orientations of this radical in the crystal lattice.

Ban et al. ${ }^{68}$ also investigated these alanine irradiation products and showed that the computed hyperfine couplings are in good agreement with experiment. These authors also performed computations on the zwitterionic forms using an Onsager solvent model. Moreover, they also concluded that the amino protons in radical R2 are fixed by intermolecular hydrogen bonding in the $\mathrm{L}-\alpha$-alanine crystals. The identity and properties of this radical will now be further examined including the introduction of the crystal environment.

\section{Effect of the crystal environment - Radical R2}

We have previously studied the effect of the molecular environment on both the geometry and the EPR hyperfine coupling constants for the radical $\mathrm{R} 2 .^{69,70}$ In order to describe the crystal environment, both cluster and periodic calculations were performed.

In the cluster calculations, a cluster model of seven and 15 alanine molecules, respectively, was constructed; the starting geometries for these optimizations were then obtained by abstracting a hydrogen atom from the central alanine molecule. The geometries of these clusters were optimized using a two-layered ONIOM model, ${ }^{71-75}$ and the level of theory at which the separate layers were treated was varied. The inner layer contained the radical and was described at either the semi-empirical PM3 level or at the DFT level, using a series of different functionals. The semiempirical PM3 and AM1 levels and the DFT level were adopted separately to treat the surrounding alanine cluster.

The periodic calculations were initiated starting from the experimental crystal structure of $\mathrm{L}-\alpha$-alanine. Next, the radical was constructed by abstracting one hydrogen atom and it was placed in a periodic supercell, obtained by doubling the unit cell in both the $a$ and $c$ directions.

From the geometric point of view, the main conclusion was that the central carbon atom of the radical deviates from planarity and that the magnitude of this deviation depends on the level of theory employed. On average, this deviation amounts to $7^{\circ}$, contrasting with previously used geometries which were considered to be perfectly planar, in line with the transformation of this carbon atom from $\mathrm{sp}^{3}$ to $\mathrm{sp}^{2}$ hybridization upon radical formation. It can therefore be invoked that the interactions with the neighbouring alanine molecules force the radical into a nonplanar conformation. This deviation from planarity appears to be a direct consequence of the appearance of hydrogen bonds, which induce rotation of the $\mathrm{CO}_{2}$ group. The methyl protons, obviously not involved in hydrogen bonding, can therefore be involved in quasi-free rotations in the crystal, as opposed to the amino protons which are involved in hydrogen bonding and thus remain relatively fixed. The strength of these hydrogen bonds determines the magnitude with which the radical deviates from planarity. It is clear from this work that the central alanine radical is forced into a conformation that would not correspond to an isolated radical equilibrium conformation by the surrounding alanine molecules.

It turns out that the partial $\mathrm{sp}^{2}$ character of the $\mathrm{C} 4$ atom has an important influence on the hfccs of the surrounding 
atoms; this non-planarity turns out to be essential for the accurate reproduction of the nitrogen hfcc in the radical. In Fig. 7, the $\mathrm{N}$ hfcc is plotted against the dihedral angle $\mathrm{C} 1-\mathrm{N} 4-\mathrm{C} 3-\mathrm{C} 2$, a measure of the non-planarity of the radical backbone.

As can be seen, the $\mathrm{N}$ hfcc shows an almost linear dependence on the deviation from planarity. The experimental value is best reproduced by the B3LYP/PM3/14 and BP86/PW geometry. This finding is confirmed by taking the geometry yielding the worst agreement with experiment for the $\mathrm{N} \mathrm{hfcc}$, and fixing this dihedral angle at $17.22^{\circ}$, the value resulting from the B3LYP/PM3/14 level. Using this geometry to recompute the $\mathrm{N}$ hfcc yields the entry in Fig. 7 indicated by an asterisk, very close to the regression line.

For the methyl group, the result is somewhat different: good agreement with experiment, as was found earlier, appears to be possible only when assuming a planar radical backbone.
In order to investigate this finding, the following procedure was performed. Starting from the B3LYP/PM3/14 geometry, the planarity of the radical was gradually increased by first reducing the dihedral angle $\mathrm{C} 1-\mathrm{N} 4-$ C3-C2 to zero, keeping all other internal coordinates fixed at their optimized values. Next, the $\mathrm{CO}_{2}$ group was gradually rotated toward a fully planar conformation of the radical backbone. During this procedure, the average methyl proton hfccs were computed at each step and are depicted in Fig. 8.

The first step induces an increase of $10 \mathrm{MHz}$ for this quantity and the second step an additional increase of $5 \mathrm{MHz}$. The remaining discrepancy from the experiment can probably be ascribed to remaining geometry parameters that were not considered. This procedure confirms the earlier findings that R2 shows a planar structure. This discrepancy with the cluster and periodic calculations is probably due to temperature effects on the geometry; thermal agitation weakens the hydrogen bonds, weakening the forces that keep

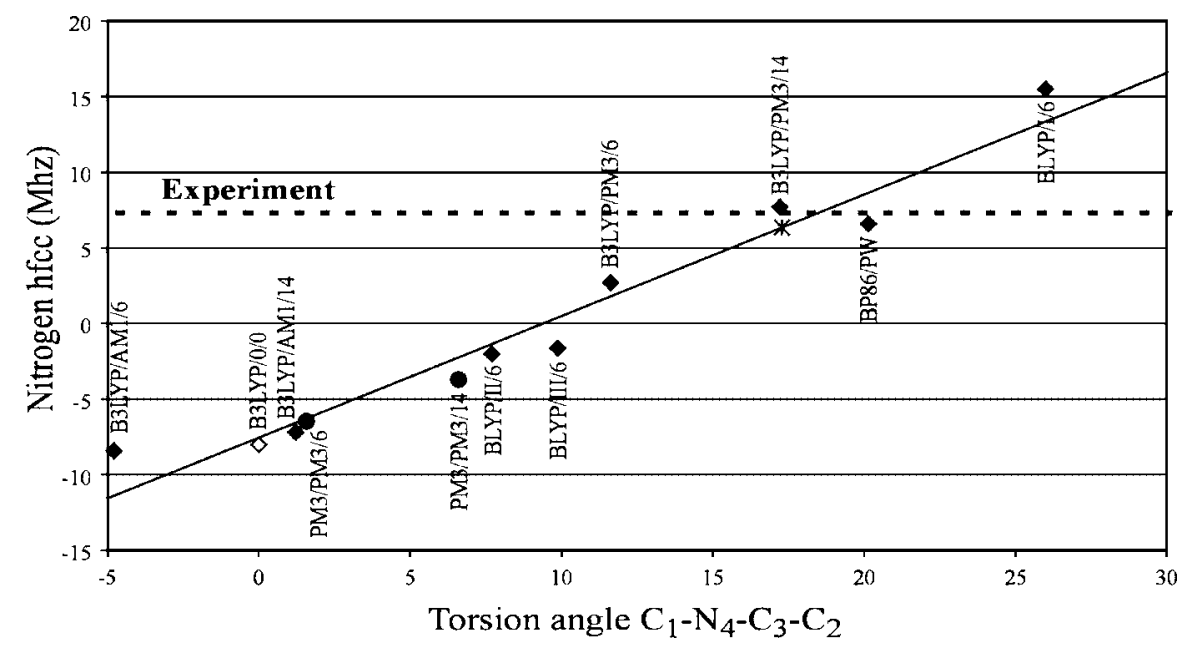

Figure 7. Plot of the nitrogen hyperfine coupling constant as a function of the $\mathrm{C} 1-\mathrm{N} 4-\mathrm{C} 3-\mathrm{C} 2$ torsion angle at different levels of theory. The dotted line represents the experimental value. For a definition of the different theoretical levels, see Refs 69 and 70. Reproduced with permission from Pauwels E et al. (Ref. 69). Copyright 2001 American Chemical Society.

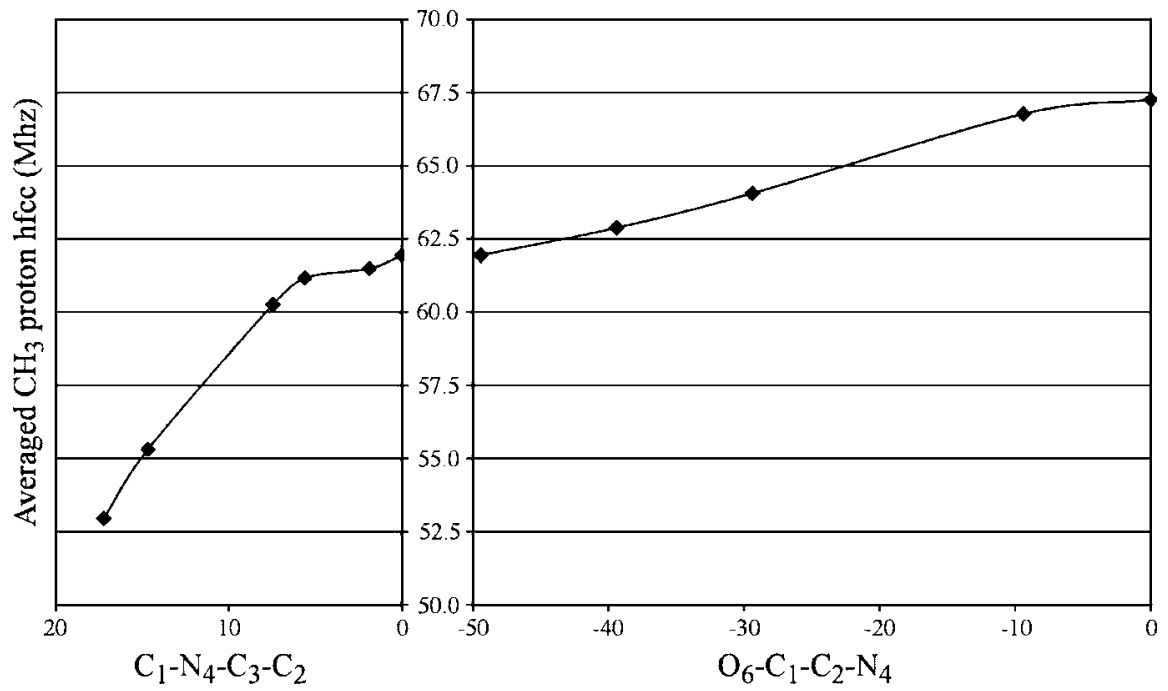

Figure 8. Plot of the averaged methyl proton hyperfine coupling constants as a function of the planarity of the radical backbone and rotation of the $\mathrm{CO}_{2}$ group. For a definition of the different theoretical levels, see Refs 69 and 70 . Reproduced with permission from Pauwels E et al. (Ref. 69). Copyright 2001 American Chemical Society. 
the central radical in a non-planar conformation. Vibrational effects can also be invoked to explain this discrepancy.

We finally focus on the amino protons. Since these are involved in intermolecular hydrogen bonds, these are relatively fixed geometrically. From Fig. 9, it can be seen that both the cluster models and periodic structures satisfactorily reproduce the $h f c c$.

In a subsequent paper, ${ }^{76}$ Petrenko performed a study on the formation of various radical structures in the irradiated L- $\alpha$-alanine crystal using a cluster consisting of 208 atoms. Geometries and relative energies were obtained at the semi-empirical PM3 level, which was found to reproduce adequately the hydrogen bond lengths in the undamaged alanine crystal in a series of test calculations. Next, a large number (20) of radicals were generated and it was shown that the radicals with the lowest energies yield EPR properties in agreement with the experimentally observed radicals. Close attention was paid to the correct description of the rotationally averaged hyperfine properties of the methyl protons.

From the isolated radical study of L- $\alpha$-alanine, it can be concluded that the experimental assignments are largely confirmed by the theoretical calculations. The proton hfccs, computed at the B3LYP/6-311G(d) level, presented there show a maximum deviation of $10 \%$ from experiment; using the larger and therefore computationally more involved IGLO III basis set yields comparable results.

It was found, however, that the accuracy of the calculated carbon and nitrogen values is more difficult to assess, mainly owing to the lack of sufficiently reliable experimental data. In some cases, however, accounting for the molecular environment, as was explicitly established in the case of radical $\mathrm{R} 2$, proved crucial for the accurate modelling of the radical geometry and was shown to yield further insight in to the observed hfccs of the radicals. In that case, the effects of vibrational averaging were shown to be of crucial importance for obtaining an acceptable agreement with experiment.

The computational strategy and methodology described are therefore a guide to investigate, on a routine basis, the structure of radiation-induced bioradicals. It also offers, when necessary, the possibility of including thermal effects and effects of the molecular environment.

\section{Learning from the case of alanine: hyperfine couplings in steroid radicals}

This methodology was also adopted and shown to be valuable in the case of a series of radiation-induced free radicals in steroid hormone crystals.

Steroid hormones are natural metabolites of cholesterol and differ from the latter in the presence of a coupled system of double bonds between the carbonyl group attached to the C3 atom and the carbon atoms C4 and C5. The androstane skeleton with the labelling of the rings and the carbon atoms is shown in Fig. 10.

The radiation-induced radicals of steroid compounds have been examined using electron paramagnetic resonance

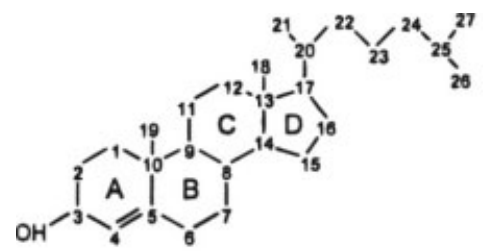

(a)

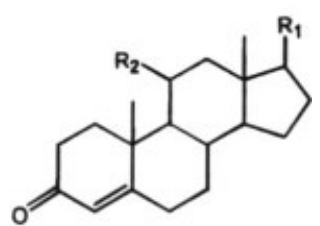

(b)
Figure 10. (a) Carbon atom labelling and ring notation for cholesterol; (b) the steroid skeleton [e.g. androgens (testosterone): $\mathrm{R} 1=\mathrm{OH}, \mathrm{R} 2=\mathrm{H}]$. Reproduced with permission from Lahorte P et al. (Ref. 3). Copyright 1999 American Chemical Society.

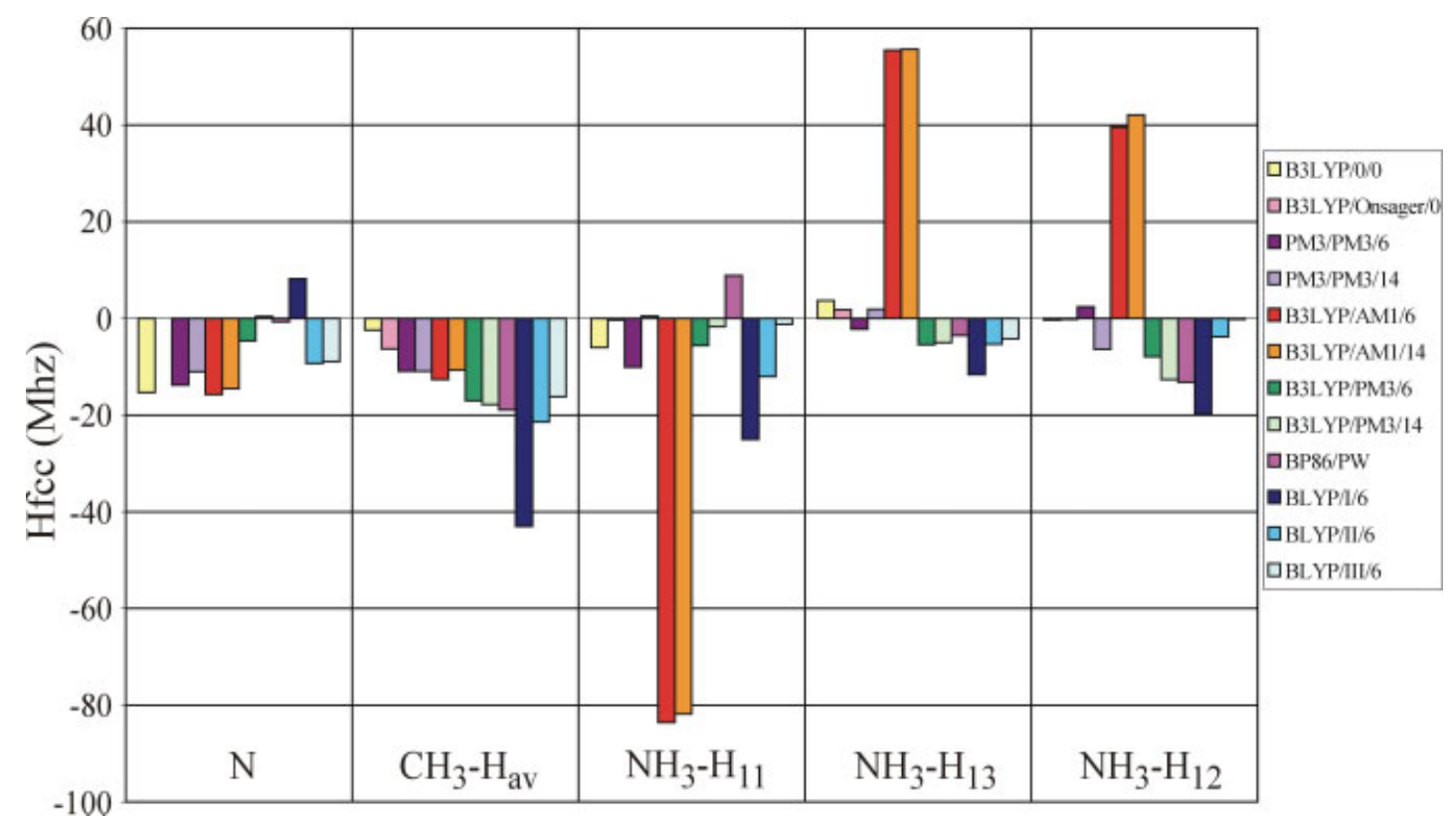

Figure 9. Overview of the different hfcc calculated at various levels of theory relative to the experimental results for the nitrogen and the methyl and amino protons. For a definition of the different theoretical levels, see Refs 69 and 70 . Reproduced with permission from Pauwels E et al. (Ref. 69). Copyright 2001 American Chemical Society. 
(EPR) and electron nuclear double resonance (ENDOR) spectroscopy. ${ }^{77-79}$ In general, these studies have led to the conclusion that the majority of radicals in irradiated steroid hormones are created in the A and B rings. More specifically, four types of radicals have been identified or suggested, ${ }^{78}$ which are displayed in Fig. 11.

For each of the radical species, a model system was constructed, by neglecting the $C$ and $D$ rings from the steroid skeleton as their influence on the hfccs can be expected to be small.

Again, for radicals R1, R2 and R3, overall good agreement between the calculated and experimental values was obtained, the differences on average being $<5 \%$. For R3, it was even suggested that the experimental assignments for two protons should be interchanged.

For radical R4 however, the agreement between the experimental and calculated hfccs was found to be poor for all atoms except one $\beta$-type coupling at a hydrogen atom. Several reasons were be invoked to explain this discrepancy.

First and foremost, of all of the model radicals considered in this section, the changes in conformation upon radical formation are probably the largest in this radical. In this species, the radical is created at a carbon atom connecting the $\mathrm{A}$ and $\mathrm{B}$ rings, whereas in the other radicals, the change in hybridization is in only one ring (R1 or R2) or even outside the rings (R3).

Another possible reason for the discrepancy is the fact that the omission of the $\mathrm{C}$ ring in this model radical structure could have greater consequences for R4 than for the other radicals. In order to check this, we constructed an extended model radical, incorporating the $\mathrm{C}$ ring, but this did not lead to a substantial improvement of the agreement with the experimental hfccs. For this radical R4, it therefore appears that the isolated molecule is partially successful. However, in our opinion, the excellent quality of the calculations, as demonstrated in the case of the radicals R1, R2 and R3, suggests, in this case, the involvement of subtle geometry effects. To resolve this issue, one should probably include nearest neighbouring atoms in the crystal lattice in the analysis, as performed and described above for the radical $\mathrm{R} 2$ of alanine.

Therefore, the possibility should be kept open that the proposed model structure for R4 is incorrect. In this case, a different radical should be put forward which more closely matches the experimental observations.

\section{TENTATIVE STRUCTURES FOR RADICALS DERIVED FROM CRYSTALLINE $\beta$-D-FRUCTOSE: THE PROBLEM OF THE EIGENVECTORS}

\section{Introduction}

In the previous section, we have shown that the computational methodology put forward in the first section performs satisfactorily in the determination of the structures of radiation-induced alanine and steroid radicals. In this final section, this methodology will be used to propose possible radical structures for the radiation-induced radicals of crystalline $\beta$-D-fructose. It should be noted that this problem is of greater complexity than the problem addressed in the previous section, where radical structures were already put forward by the experimentalists. It will indeed turn out that, in order to provide an acceptable answer as to the nature and the structure of these radiation-induced radicals, a detailed analysis of the eigenvectors of the proton hyperfine tensors had to be made.

In recent years, there has been considerable interest in the radiation-induced radicals in sugars, in view of the radiation treatment of sugar-containing food. This has led to research into the development of dosimetric protocols for the various foodstuffs that are eligible for radiation treatment. In this respect, the radiation chemistry of sugars is enjoying ever-increasing interest because of the stability of radiationinduced sugar radicals that consequently might represent suitable probes for the detection of irradiated, sugar-rich foodstuffs using EPR spectroscopy.

It was only recently that an EPR study was conducted on irradiated solid-state $\beta$-D-fructose using ENDOR and ENDOR-induced EPR (EI-EPR). Vanhaelewyn et al. performed a combined experimental and theoretical study on the EPR properties of irradiated D-fructose crystals. ${ }^{5}$ In their contribution, the identification of two dominant radicals, which will be indicated as F1 and F2, is reported. ${ }^{5}$ Both of these radicals are characterized by three $\beta$-type hyperfine couplings, as detected by the EI-EPR experiment, given in Table 3. This suggests that the unpaired electron of both the fructose radicals interacts with three protons yielding six hydrogen hyperfine tensors. However, only five tensors have been determined with ENDOR, the missing tensor in F2 probably corresponding to a small hyperfine interaction that could not be determined unambiguously.

On the basis of the experimental data and theoretical considerations, several model fructose radicals can be suggested.

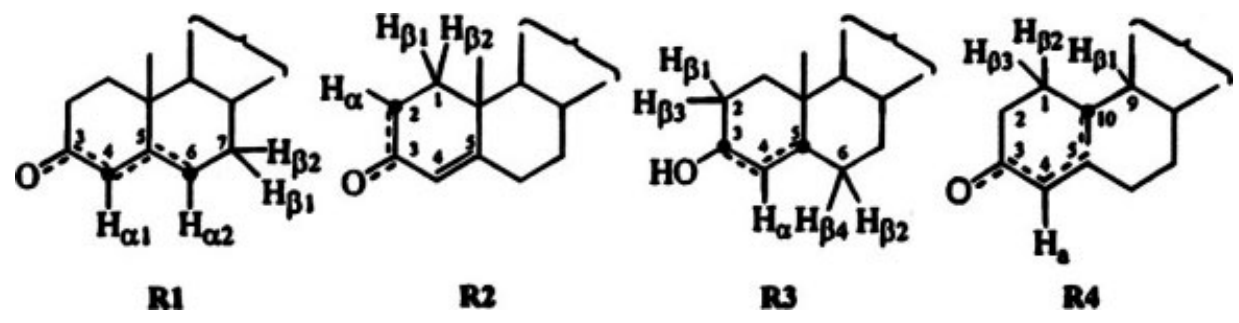

Figure 11. Schematic overview of the four types of radicals formed in irradiated crystals of steroids. Reproduced with permission from Lahorte P et al. (Ref. 3). Copyright 1999 American Chemical Society. 
Table 3. Experimental hfcc values and direction cosines of radicals $F_{1}$ and $F_{2}$, taken from our previous work. ${ }^{77}$ Reproduced with permission from Pauwels E et al. (Ref. 4). Copyright 2002 American Chemical Society

\begin{tabular}{lrrrrrr}
\hline & \multicolumn{5}{c}{ Experiment } \\
\cline { 2 - 7 } Coupling & $A_{\text {iso }}$ & $T_{\text {aniso }}$ & \multicolumn{1}{c}{$A$} & & Axes \\
\hline F1- $\beta 1$ & 96.9 & -4.2 & 92.7 & -0.448 & -0.253 & 0.858 \\
& & -2.9 & 94.0 & 0.675 & -0.724 & 0.139 \\
& & 7.1 & 104.0 & 0.586 & 0.642 & 0.495 \\
F1- $\beta 2$ & 37.3 & -4.1 & 33.2 & -0.476 & 0.86 & 0.185 \\
& & -2.4 & 34.9 & -0.78 & -0.51 & 0.362 \\
& & 6.6 & 43.9 & 0.406 & 0.028 & 0.914 \\
F1- $\beta 3$ & 9.3 & -3.3 & 6.0 & -0.318 & -0.946 & -0.066 \\
& & -1.7 & 7.6 & -0.225 & 0.008 & 0.974 \\
& & 5.0 & 14.3 & -0.921 & 0.325 & -0.215 \\
F2- $\beta 1$ & 87.5 & -3.6 & 83.9 & -0.479 & -0.365 & 0.798 \\
& & -3.2 & 84.3 & 0.642 & -0.766 & 0.035 \\
& & 6.8 & 94.3 & 0.598 & 0.529 & 0.601 \\
F2- $\beta 2$ & 43.1 & -3.9 & 39.2 & -0.262 & 0.929 & 0.263 \\
& & -2.7 & 40.4 & -0.852 & -0.351 & 0.389 \\
& & 6.5 & 49.6 & 0.453 & -0.122 & 0.883 \\
& & & & & &
\end{tabular}

In this section, we propose and investigate four model structures depicted in Fig. 12, all being serious candidates for the experimentally observed fructose radicals. ${ }^{4}$

Radical $\mathrm{F}_{\mathrm{A}}$ is constructed by $\mathrm{OH}$ group abstraction from C5; this yields a structure with no $\alpha$-type proton. Moreover, the unpaired electron is presumably delocalized over C5 and O1. Furthermore, at least three protons are suitably located to produce a $\beta$-type coupling with the radical centre: these are the protons of the hydroxy methyl group at C6, the proton at $\mathrm{C} 4$ and, through the probable delocalization over the ring oxygen, both protons at $\mathrm{C} 1$. A second set of possible radicals is obtained with the extraction of a hydrogen atom from the undamaged $\beta$-D-fructose structure. Two structures, $\mathrm{F}_{\mathrm{B}}$ and $\mathrm{F}_{\mathrm{C}}$, where a hydrogen is abstracted from either $\mathrm{C} 2$ or $\mathrm{C} 3$, were further investigated owing to their potential consistency with the experimental observations. In $\mathrm{F}_{\mathrm{B}}$, both $\mathrm{C} 1$ protons, the hydroxy proton at $\mathrm{O} 2$ and the proton at $\mathrm{C} 3$ can produce a $\beta$-type coupling with the unpaired electron, whereas in the model structure $\mathrm{F}_{\mathrm{C}}$, the protons at $\mathrm{C} 2$ and $\mathrm{C} 4$, together with the hydroxy proton at $\mathrm{O} 3$ are in a $\beta$-position relative to the radical centre. The fourth model radical structure that was investigated, $\mathrm{F}_{\mathrm{D}}$, was generated by the abstraction of the hydroxymethyl group from C5. In this structure, the unpaired electron will, most probably, be delocalized over the ring oxygen $\mathrm{O} 1$ and carbon $\mathrm{C} 5$. As a result, four possible $\beta$-type couplings can be generated in this structure by the ring protons $\mathrm{H} 1 \mathrm{~A}$ and $\mathrm{H} 1 \mathrm{~B}$, through the delocalization of the radical centre, together with the ring proton $\mathrm{H} 4$ and the hydroxy proton $\mathrm{HO} 4$.

In the next section, radical $\mathrm{F}_{\mathrm{A}}$ will be investigated in full detail to assess its value in reproducing the experimentally observed coupling characteristics. For the model structures $F_{B}$ and $F_{C}$, investigation of the isotropic and anisotropic hyperfine coupling constants and the comparison of the
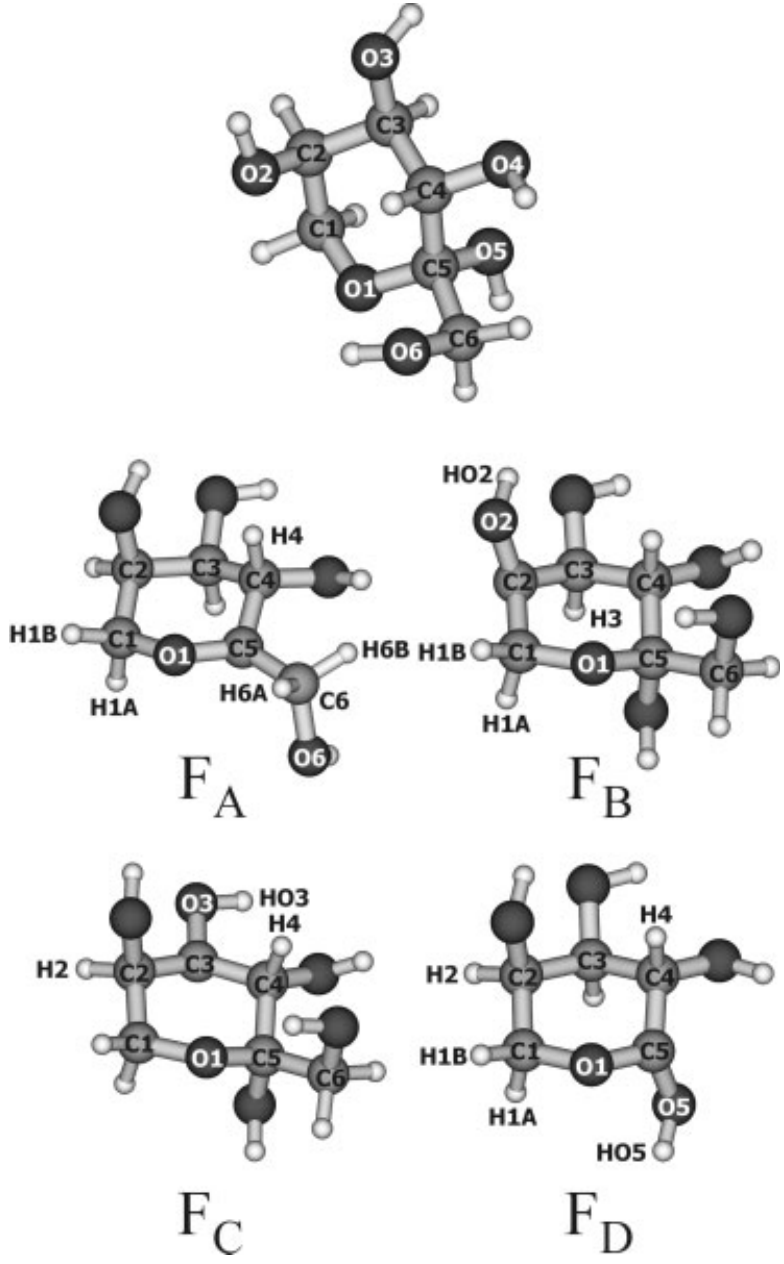

Figure 12. Molecular structure of $\beta$-D-fructose as determined by neutron diffraction, together with the optimized geometries of the proposed model radicals. Reproduced with permission from Pauwels E et al. (Ref. 4). Copyright 2002 American Chemical Society.

angles of the calculated proton tensor components and the experimental ones revealed that these model radicals are not realistic candidates to be identified as either $F_{1}$ or $F_{2}$. Also, model radical $F_{D}$ was found to be not consistent with the experimental results.

\section{Model structure $\mathbf{F}_{\mathrm{A}}$}

In the optimized radical structure, the unpaired spin turned out to be mainly located at C5 and to a smaller extent at $\mathrm{O} 1$, the spin densities being 0.83 and 0.12 , respectively. In addition, it can be noted that the $\mathrm{sp}^{3}$ hybridization of $\mathrm{C} 5$ is partially retained with respect to the undamaged fructose molecule.

The most relevant degree of freedom of this radical is the internal rotation of the hydroxy methyl group about the C5-C6 single bond, characterized by the dihedral angle $\mathrm{O} 6-\mathrm{C} 6-\mathrm{C} 5-\mathrm{C} 4$; the results of the variation of this angle are given in Fig. 13. Also displayed in Fig. 13 are the experimentally measured isotropic hfccs.

As can be seen and as could be expected, the largest variation occurs for the protons H6A and H6B. The large $\mathrm{F} 1-\beta 1$ coupling of $96.6 \mathrm{MHz}$ most likely corresponds to the $\mathrm{H} 4 \mathrm{hfcc}$. The H1A and H1B proton hfcc values remain 


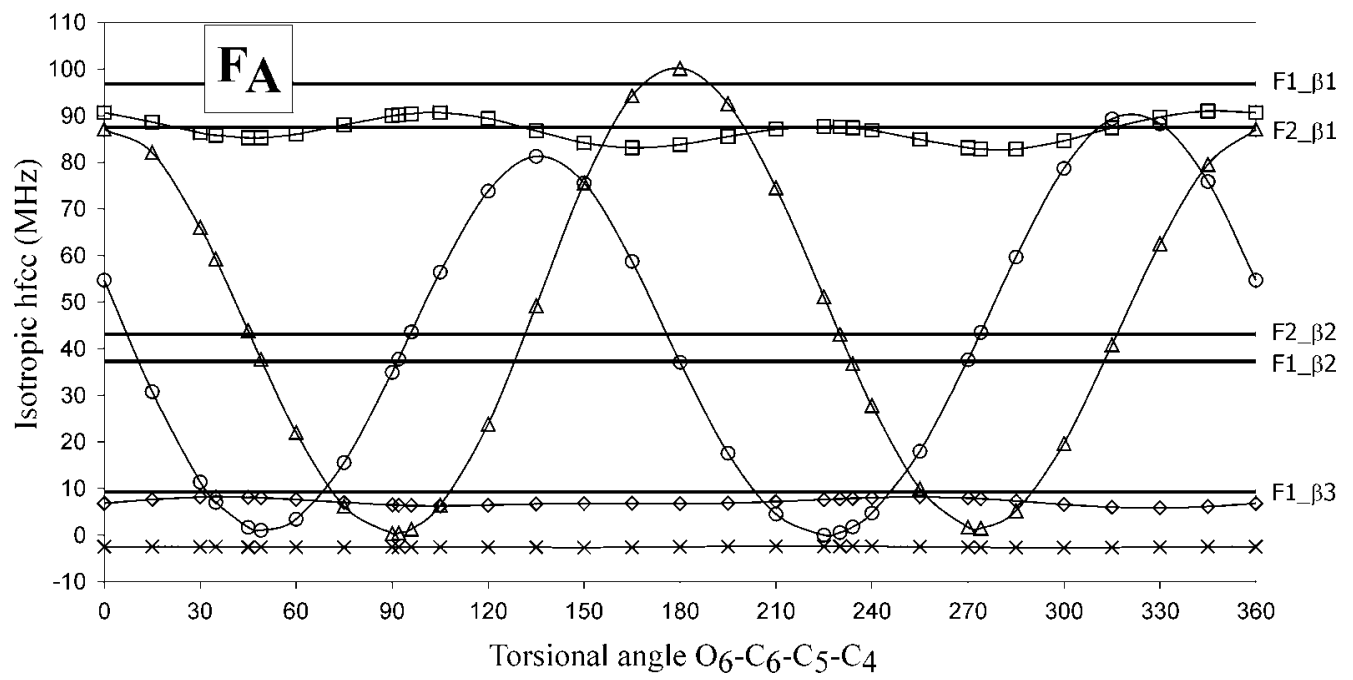

Figure 13. Isotropic proton hfccs in model radical $F_{A}$ as a function of the hydroxymethyl group rotation $[(\bullet) H 1 A ;(\times) H 1 B ;(\square) H 4 ;(\triangle)$ $\mathrm{H} 6 \mathrm{~A}$; (O) H6B; all other proton hfcc's were close to zero throughout the rotation and are therefore not included]. Solid lines indicate the experimental hfcc values. Reproduced with permission from Pauwels E et al. (Ref. 4). Copyright 2002 American Chemical Society.

practically constant throughout the rotation, but the H1B proton displays a smaller coupling then H1A. If one assigns the H1A hfcc to the experimental F1- $\beta 3$ coupling $(9.3 \mathrm{~Hz})$, it is clear that one of the hydroxy methyl proton hfcc values should correspond to the $\mathrm{F} 1-\beta 2$ coupling $(37.3 \mathrm{MHz})$ whereas the other should be close to zero and consequently not detectable experimentally. From Fig. 13, it can be invoked that only four conformations succeed in reproducing the three experimental signals of F1 theoretically:

1. A torsional angle of $49^{\circ}$ yields an H6A hfcc of $37.8 \mathrm{MHz}$, corresponding to the experimental F1- $\beta 2$ coupling of 37.3 MHz.

2. A torsional angle of $92^{\circ}$ yields an hfcc of $37.8 \mathrm{MHz}$ for H6B, in agreement with experiment.

3. At an angle of $234^{\circ}$, the H6A hfcc amounts to $37.6 \mathrm{MHz}$.

4. At an angle of $270^{\circ}$, the H6B hfcc yields $37.6 \mathrm{MHz}$.

In addition, it should be mentioned that, for all of the four cases outlined above, the hfcc of the counterpart proton (H6A or $\mathrm{H} 6 \mathrm{~B}$ ) is below $6 \mathrm{MHz}$, in agreement with the fact that these signals have not been detected experimentally.

In order to gain more insight into and to disentangle the four conformations proposed above, a detailed study was made of the anisotropic components of the hyperfine tensor and their corresponding spatial directions. It turned out, however, that the anisotropic components of the hyperfine tensor were all in close agreement with the experimental results and did not differ significantly from each other. In order to determine the conformation that corresponds with reality, an analysis was performed of the direction cosines of the different associated eigenvectors. To the best of our knowledge, this is the first analysis of this type for radicals occurring in organic crystals.

The three principal axes of the experimental hyperfine tensors are specified by the direction cosines with respect to some reference frame, which usually coincides with the crystal axes. However, since the theoretical analysis did not simulate the crystal environment, it is impossible to insert this reference axis system as was done in the experiments. In order to proceed and to solve this ambiguity, the angles between the experimental principal axes were compared with the angles between the calculated proton tensor axes, as shown in Fig. 14 for the $\beta 1$ and $\beta 3$ signals in radical F1. In this way, the choice of the reference axis system is eliminated and one obtains an additional reliable tool to differentiate between the four conformations proposed by theory.

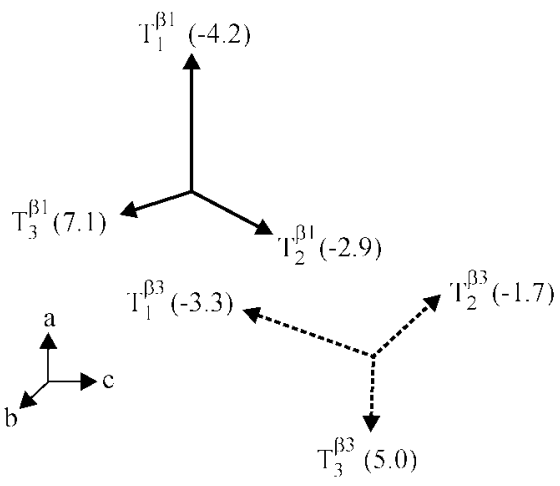

(a)

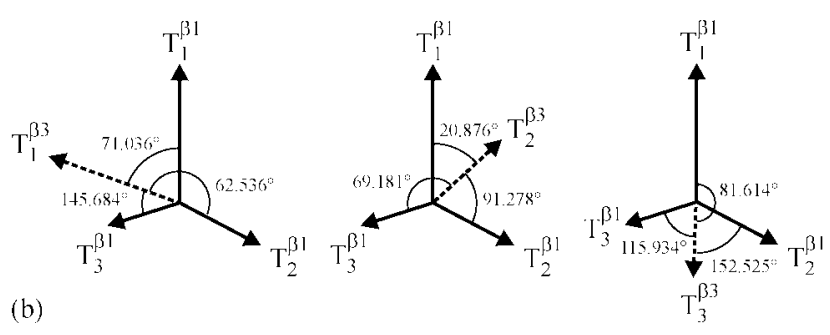

Figure 14. (a) Principal axes of the experimental $\beta 1$ and $\beta 3$ signals as given by their direction cosines with respect to the $a b c$ reference frame. (b) Angles between the principal axes of the hyperfine tensor; the principal angles of the hyperfine tensors are specified by their corresponding anisotropic values $(\mathrm{MHz})$. Reproduced with permission from Pauwels $\mathrm{E}$ et al. (Ref. 4). Copyright 2002 American Chemical Society. 
When analysing the results, however, it should be stressed that there still remains some ambiguity in fixing the relative angles because the absolute sign of the experimental direction cosines cannot be determined. This ambiguity was taken into consideration when analysing the relative angles, which are reported in Table 4, together with their calculated counterparts.

As can be seen from Table 4, the angles between the principal axes, calculated for the conformation at $234^{\circ}$, are in close agreement with the angles between the experimental $F_{1}$ signals. The other conformations, however, do not succeed by far in reproducing the experimental findings. It should be noted that the smallest two anisotropic components for the $\mathrm{H} 4$ proton have been switched to allow a fair comparison. This means that for the conformation at $234^{\circ}$, the eigenvector for the $-2.0 \mathrm{MHz}$ anisotropic component of $\mathrm{H} 4$ matches the eigenvector of the $-4.2 \mathrm{MHz}$ experimental component of $\mathrm{F}_{1}$, and that the $-5.3 \mathrm{MHz}$ eigenvector of $\mathrm{H} 4$ can be identified with the $-2.9 \mathrm{MHz} \mathrm{F} 1$ experimental component. The reason for this switch between the low anisotropic component axes can be attributed to the fact that the present calculations did not take into account the effect of the molecular environment, which, of course, should be confirmed by performing larger cluster calculations. Apart from this interchange, all anisotropic and isotropic proton hyperfine couplings in the experimental species $\mathrm{F}_{1}$ are in close agreement with the values of $\mathrm{F}_{\mathrm{A}}$, as summarized in Table 5. These results were confirmed using the larger EPR-III basis set. Moreover, the fair reproduction of the mutual angles between the anisotropic principal axes strengthens the identification of the experimental species $\mathrm{F}_{1}$ as having a radical structure in agreement with $\mathrm{F}_{\mathrm{A}}$, and with a hydroxy methyl torsional angle of $234^{\circ}$ for $\mathrm{O} 6-\mathrm{C} 6-\mathrm{C} 5-\mathrm{C} 4$.

These findings support the conclusion that the F1 and F2 species are in fact manifestations of the same radical, with a structure conform $\mathrm{F}_{\mathrm{A}}$, but with slightly altered conformations. On the basis of the comparison with the experimental isotropic values, only minor conformational changes are found (about $4^{\circ}$ ), which are not of sufficient extent to differentiate further between radical species F1 and F2, based on the single-molecule approach. It should be mentioned, however, that experimental information is partially lost in this approach since crystal axes are not involved in the model. Moreover, translational and rotational degrees of freedom of the radical within the crystal lattice are

Table 4. Comparison of the angles between the calculated tensor components of the $F_{A}$ protons (calculated for a torsional angle of $234^{\circ}$ ) and the angles between the experimental tensor components. Reproduced with permission from Pauwels E et al. (Ref. 4). Copyright 2002 American Chemical Society

\begin{tabular}{|c|c|c|c|c|c|c|c|c|c|c|c|}
\hline \multicolumn{6}{|c|}{ Experiment } & \multicolumn{6}{|c|}{ Theory } \\
\hline \multirow{9}{*}{$\mathrm{F} 1-\beta 3$} & & \multicolumn{4}{|c|}{$\mathrm{F} 1-\beta 1$} & \multirow{9}{*}{$\mathrm{H} 1 \mathrm{~A}$} & \multirow{3}{*}{$\overline{A_{\text {iso }}}$} & & \multicolumn{3}{|c|}{$\mathrm{H} 4$} \\
\hline & \multirow[t]{2}{*}{$\overline{A_{\text {iso }}}$} & \multicolumn{4}{|c|}{96.9} & & & \multicolumn{4}{|c|}{87.4} \\
\hline & & $T_{\text {aniso }}$ & -4.2 & -2.9 & 7.1 & & & $T_{\text {aniso }}$ & -2.0 & -5.3 & 7.3 \\
\hline & & -3.3 & 71.0 & 62.5 & 145.7 & & & -4.3 & 84.2 & 75.4 & 164.3 \\
\hline & 9.3 & -1.7 & 20.9 & 91.3 & 69.2 & & 7.9 & -2.9 & 10.1 & 83.4 & 82.3 \\
\hline & & 5.0 & 81.6 & 152.5 & 115.9 & & & 7.2 & 81.7 & 164.0 & 103.6 \\
\hline & & \multicolumn{4}{|c|}{$\mathrm{F} 1-\beta 1$} & & & & \multicolumn{3}{|c|}{$\mathrm{H} 4$} \\
\hline & $\overline{A_{\text {iso }}}$ & \multicolumn{4}{|c|}{96.9} & & $\overline{A_{\text {iso }}}$ & & \multicolumn{3}{|c|}{87.4} \\
\hline & & $T_{\text {aniso }}$ & -4.2 & -2.9 & 7.1 & & & $T_{\text {aniso }}$ & -2.0 & -5.3 & 7.3 \\
\hline \multirow{3}{*}{$\mathrm{F} 1-\beta 2$} & & -4.1 & 81.1 & 156.7 & $\overline{68.6}$ & \multirow{3}{*}{ H6A } & & -4.7 & 76.4 & 126.0 & 39.3 \\
\hline & 37.3 & -2.4 & 37.9 & 96.1 & 127.2 & & 36.8 & -3.0 & 35.4 & 108.3 & 119.1 \\
\hline & & 6.6 & 53.5 & 67.6 & 44.9 & & & 7.7 & 58.1 & 41.8 & 66.1 \\
\hline \multirow{9}{*}{$\mathrm{F} 1-\beta 3$} & & \multicolumn{4}{|c|}{$\mathrm{F} 1-\beta 2$} & \multirow{12}{*}{ H1A } & & & \multicolumn{3}{|c|}{ H6A } \\
\hline & $\overline{A_{\text {iso }}}$ & & & 37.3 & & & \multirow{3}{*}{$A_{\text {iso }}$} & & \multicolumn{3}{|c|}{36.8} \\
\hline & & $T_{\text {aniso }}$ & -4.1 & -2.4 & $\overline{6.6}$ & & & $\overline{T_{\text {aniso }}}$ & -4.7 & -3.0 & 7.7 \\
\hline & & -3.3 & 132.4 & 45.0 & $\overline{102.4}$ & & & -4.3 & 125.0 & 51.0 & 121.6 \\
\hline & 9.3 & -1.7 & 72.9 & 58.4 & 37.0 & & 7.9 & -2.9 & 66.2 & 39.3 & 60.7 \\
\hline & & 5.0 & 47.3 & 61.6 & 124.1 & & & 7.2 & 44.5 & 86.0 & 134.2 \\
\hline & & \multicolumn{4}{|c|}{$\mathrm{F} 2-\beta 1$} & & & & & & \\
\hline & $\overline{A_{\text {iso }}}$ & \multicolumn{4}{|c|}{87.5} & & & & & & \\
\hline & \multirow{4}{*}{43.1} & $\overline{T_{\text {aniso }}}$ & -3.6 & -3.2 & $\overline{6.8}$ & & & & & & \\
\hline \multirow{3}{*}{$\mathrm{F} 2-\beta 2$} & & -3.9 & 90.2 & 150.5 & 60.5 & & & & & & \\
\hline & & -2.7 & 32.1 & 105.3 & 117.5 & & & & & & \\
\hline & & 6.5 & 57.8 & 65.5 & 42.5 & & & & & & \\
\hline
\end{tabular}


Table 5. Summary of calculated proton hyperfine tensor components for the $F_{A}$ conformation with a torsional angle of $234^{\circ}$. Reproduced with permission from Pauwels E et al. (Ref. 4). Copyright 2002 American Chemical Society

\begin{tabular}{|c|c|c|c|c|c|c|c|c|}
\hline & \multicolumn{7}{|c|}{ Conformation at $234^{\circ}$} & \multirow{3}{*}{$\begin{array}{l}\text { Experimental } \\
\text { match }\end{array}$} \\
\hline & \multicolumn{3}{|c|}{$6-311 G^{*}$} & \multicolumn{3}{|c|}{ EPR-III } & \multirow[b]{2}{*}{$\Delta_{\text {direction }}$} & \\
\hline & $A_{\text {iso }}$ & $T_{\text {aniso }}$ & $A$ & $A_{\text {iso }}$ & $T_{\text {aniso }}$ & $A$ & & \\
\hline \multirow[t]{3}{*}{ H1A } & 7.9 & -4.3 & 3.6 & 8.5 & -4.4 & 4.1 & 1.489 & \multirow{3}{*}{$\mathrm{F} 1-\beta 3$} \\
\hline & & -2.9 & 5.0 & & -2.7 & 5.8 & 1.495 & \\
\hline & & 7.2 & 15.1 & & 7.1 & 15.6 & 0.172 & \\
\hline \multirow[t]{3}{*}{ H1B } & \multirow[t]{3}{*}{-2.4} & -3.1 & -5.5 & -2.7 & -3.0 & -5.7 & 5.348 & \\
\hline & & -2.6 & -5.0 & & -2.5 & -5.2 & 5.352 & \\
\hline & & 5.7 & 3.3 & & 5.6 & 2.9 & 0.183 & \\
\hline \multirow[t]{3}{*}{$\mathrm{H} 4$} & \multirow[t]{3}{*}{87.4} & -2.0 & 85.4 & 94.9 & -2.2 & 92.7 & 3.656 & \multirow{3}{*}{$\mathrm{F} 1-\beta 1$} \\
\hline & & -5.3 & 82.1 & & -5.2 & 89.7 & 3.652 & \\
\hline & & 7.3 & 94.7 & & 7.4 & 102.3 & 0.343 & \\
\hline \multirow[t]{3}{*}{ H6A } & \multirow[t]{3}{*}{36.8} & -4.7 & 32.1 & 39.5 & -4.8 & 34.7 & 1.002 & \multirow{6}{*}{$\mathrm{F} 1-\beta 2$} \\
\hline & & -3.0 & 33.8 & & -2.9 & 36.6 & 1.026 & \\
\hline & & 7.7 & 44.5 & & 7.7 & 47.2 & 0.212 & \\
\hline \multirow[t]{3}{*}{ H6B } & \multirow[t]{3}{*}{1.7} & -5.1 & -3.4 & 2.0 & -5.3 & -3.3 & 4.972 & \\
\hline & & -4.0 & -2.3 & & -4.0 & -2.0 & 4.978 & \\
\hline & & 9.1 & 10.8 & & 9.3 & 11.3 & 0.285 & \\
\hline
\end{tabular}

a The $A_{\text {iso }}, T_{\text {aniso }}$ and $A$ values (in MHz) are reported for a $6-311 \mathrm{G}^{*}$ and an EPR-III basis. No direction cosines are given, but anisotropic values are ordered in comparison with their experimental counterparts in Table $3 . \Delta_{\text {direction }}$ is the difference (in degrees) between the anisotropic principal axes calculated with both basis sets

not present and also possible vibrational averaging effects related to pyramidal inversion at the radical centre are not taken into account. We therefore conclude that both radical species F1 and F2, found in the experiments of Vanhaelewyn et al. ${ }^{5}$ can be identified as having the radical structure $\mathrm{F}_{\mathrm{A}}$, possibly with a $\mathrm{O} 6-\mathrm{C} 6-\mathrm{C} 5-\mathrm{C} 4$ torsional angle at about $234^{\circ}$.

\section{GENERAL CONCLUSIONS}

In this contribution, the use of density functional methods for the structure elucidation of radiation-induced bioradicals by comparison of computed and experimental EPR properties was discussed. From the case studies investigated (radiationinduced radicals of the amino acid alanine, steroid hormones and $\beta$-D-fructose), it could be concluded that from the methodological point of view, the choice of the B3LYP functional with the $6-311 G(d)$ or $6-311 G(d, p)$ basis sets on average results in proton hyperfine couplings with a maximum deviation of about $10 \%$ from the experimental values.

It has been shown the calculated coupling constants support the experimental assignment of the observed radicals in a number of cases such as the alanine radicals and the steroid radicals R1 and R2. In some cases, such as two protons in steroid radical R3 and for the steroid radical $\mathrm{R} 4$, agreement with experiment was shown to be far from trivial. Furthermore, tentative structures for the radical occurring in irradiated fructose have been put forward and carefully compared with experimental findings. In this case, in addition to the study of the isotropic and anisotropic components of the hyperfine tensor, the direction cosines of the principal axes of this tensor also needed to be investigated in great detail.

In some cases, however, in order to obtain good agreement with the experimental findings, the complexity of the molecular model used in the computations has to be increased, introducing the environment of the radical in the crystal. This methodology was described in the case of the radical $\mathrm{R} 2$ of alanine.

Overall, one can state that DFT calculations of isotropic and anisotropic hyperfine coupling constants and, if necessary, analysis of the hyperfine tensor eigenvectors represent a powerful tool to aid experimentalists in the elucidation of the contributions of various radicals to the observed EPR spectra. It allows for a systematic procedure to identify radiationinduced bioradicals, providing the possibility of introducing the effects of the molecular or crystal environment.

\section{REFERENCES}

1. McLaughlin WL, Taylor DM (eds). ESR Dosimetry and Applications: Proceedings of the 4th International Symposium, Published in Appl. Radiat. Isoto. 47; 1151-1687; 1996.

2. Lahorte $P$, De Proft F, Vanhaelewyn G, Masschaele B, Cauwels P, Callens F, Geerlings P, Mondelaers W. J. Phys. Chem. A 1999; 103: 6650.

3. Lahorte P, De Proft F, Callens F, Geerlings P, Mondelaers W. J. Phys. Chem. A 1999; 103: 11130.

4. Pauwels E, Lahorte P, Vanhaelewyn G, Callens F, De Proft F, Geerlings P, Waroquier M. J. Phys. Chem. A 2002; 106: 12340.

5. Vanhaelewyn G, Lahorte P, De Proft F, Mondelaers W, Geerlings P, Callens F. Phys. Chem. Chem. Phys. 2001; 3: 1729.

6. Improta R, Barone V. Chem. Rev. 2004; 104: 1231.

7. Gordy W. Theory and Applications of Electron Spin Resonance. Techniques of Chemistry, vol. XV. Wiley: New York, 1980.

8. Atherton NM. Principles of Electron Spin Resonance. Ellis Horwood: Chichester, 1993. 
9. Weil JA, Bolton JR, Wertz JE. Electron Paramagnetic Resonance, Elementary Theory and Practical Applications. Wiley: New York, 1994.

10. Atkins PW, Friedman RS. Molecular Quantum Mechanics (3rd edn). Oxford University Press: New York, 1997.

11. Meyer W. J. Chem. Phys. 1969; 51: 5149.

12. Ellinger Y, Rassat A, Subra R, Berthier G. J. Chem. Phys. 1975; 62: 1 .

13. Ellinger Y, Subra R, Levy B, Millie P, Berthier G. J. Chem. Phys. 1975; 62: 10.

14. Hilbert A. Rep. Prog. Phys. 1975; 38: 1217.

15. Sekino H, Bartlett RJ. J. Chem. Phys. 1985; 82: 4225.

16. Chipman DM. Theor. Chim. Acta 1989; 76: 73.

17. Engels B, Peyerimhoff SD. Mol. Phys. 1989; 67: 583.

18. Karna SP, Grein F, Engels B, Peyerimhoff SD. Int. J. Quantum Chem. 1989; 36: 255.

19. Karna SP, Grein F, Engels B, Peyerimhoff SD. Mol. Phys. 1990; 69: 549.

20. Carmichael I. J. Phys. Chem. 1991; 95: 6198.

21. Engels B. Chem. Phys. Lett. 1991; 179: 398.

22. Feller D, Davidson ER. In Theoretical Models of Chemical Bondings, Part 3, Maksic ZB (ed). Springer: Berlin, 1991; 429.

23. Feller D, Glendening ED, McCullough EA, Miller RJ. J. Chem. Phys. 1991; 99: 2829.

24. Perera SA, Watts JD, Bartlett RJ. J. Chem. Phys. 1994; 100: 1425.

25. Barone V, Michino C, Faucher H, Subra R, Grand A. Chem. Phys. Lett. 1993; 205: 324.

26. Eriksson LA, Lunell S, Boyd RJ. J. Am. Chem. Soc. 1993; 115: 6896.

27. Barone V, Adamo C, Russo N. Chem. Phys. Lett. 1993; 212: 5.

28. Eriksson LA, Malkin VG, Malkina OL, Salahub DR. J. Chem. Phys. 1994; 100: 5066.

29. Eriksson LA, Malkin VG, Malkina OL, Salahub DR. Int. J. Quantum Chem. 1994; 52: 879.

30. Eriksson LA, Wang J, Boyd RJ, Lunell S. J. Phys. Chem. 1994; 98 : 792.

31. Barone V. J. Chem. Phys. 1994; 101: 6834.

32. Austen MA, Eriksson LA, Boyd RJ. Can. J. Chem. 1994; 72: 695.

33. Adamo C, Barone V, Fortunelli A. J. Chem. Phys. 1995; 102: 384.

34. Barone V. In Recent Advances in Density Functional Methods, Part 1, Chong DP (ed). World Scientific: Singapore, 1995; 278.

35. Barone V. Theor. Chim. Acta 1995; 91: 113.

36. Barone V, Adamo C, Grand A, Jolibois F, Brunel Y, Subra R. J. Am. Chem. Soc. 1995; 117: 12618.

37. Malkin VG, Malkina OL, Eriksson LA, Salahub DR. In Modern Density Functional Theory, a Tool For Chemistry, Seminario JM, Politzer P (eds). Elsevier: Amsterdam, 1995; 274.

38. Martell JM, Eriksson LA, Goddard JD. Acta Chem. Scand. 1995; 51: 229.

39. Barone V, Subra R. J. Chem. Phys. 1996; 104: 2630.

40. Batra R, Giese B, Spichty M, Gescheidt G, Houk KN. J. Phys. Chem. 1996; 100: 18371

41. Zuilhof H, Dinnocenzo JP, Reddy AC, Shaik S. J. Phys. Chem. 1996; 100: 15774.

42. Gauld JW, Eriksson LA, Radom L. J. Phys. Chem. A 1997; 101: 1352.
43. Adamo C, Cossi M, Barone V. J. Mol. Struct. (Theochem) 1999; 493: 145 .

44. Knight LB, Kaup JG, Petzold B, Ayyad R, Ghanty TK, Davidson ER. J. Chem. Phys. 1999; 110: 5658.

45. Munzarova M, Kaupp M. J. Phys. Chem. A 1999; 103: 9966.

46. Pople JA, Head-Gordon M, Raghavachari K. J. Chem. Phys. 1987; 87: 5968.

47. Becke AD. J. Chem. Phys. 1993; 98: 5648.

48. Lee C, Yang W, Parr RG. Phys. Rev. B 1988; 37: 785.

49. Hehre WJ, Radom L, Schleyer PvR, Pople JA. Ab Initio Molecular Orbital Theory. Wiley: New York, 1986.

50. Kutzelnigg W, Fleisher U, Schindler M. In NMR-Basic Principles and Progress, vol. 23. Springer: Heidelberg, p 1651999.

51. Koch W, Holthausen MC. A Chemists's Guide to Density Functional Theory (2nd edn). Wiley-VCH: Weinheim, 2001.

52. Iwasaki M, Noda S, Toriyama K. Mol. Phys. 1970; 18: 201.

53. Kuroda S, Miyagawa I. J. Chem. Phys. 1982; 76: 3933.

54. Miyagawa I, Itoh K. J. Chem. Phys. 1962; 36: 2157.

55. Matsuki K, Miyagawa I. J. Chem. Phys. 1982; 76: 3945.

56. Itoh K, Miyagawa I. J. Mol. Struct. 1988; 190: 85.

57. Miyagawa I, Gordy W. J. Chem. Phys. 1960; 32: 255.

58. Morton JR, Horsfield A. J. Chem. Phys. 1961; 35: 1142.

59. Horsfield A, Morton JR, Whiffen DH. Mol. Phys. 1962; 4: 425.

60. Horsfield A, Morton JR, Whiffen DH. Mol. Phys. 1962; 5: 115.

61. Sinclair JW, Hanna MW. J. Phys. Chem. 1967; 71: 84.

62. Sinclair JW, Hanna MW. J. Chem. Phys. 1969; 50: 2125.

63. Muto H, Iwasaki I. J. Chem. Phys. 1973; 59: 4821.

64. Sagstuen E, Hole E, Haugedal S, Nelson W. J. Phys. Chem. A 1997; 101: 9763.

65. Heydari MZ, Malinen E, Hole EO, Sagstuen E. J. Phys. Chem. A 2002; 106: 8971 .

66. Malinen E, Heydari MZ, Sagstuen E, Hole EO. Radiat. Res. 2003; 159: 23.

67. Deigen MF, Krivenko VG, Pulatova MK, Ruban MA, Teslenko VV, Kayushin LPs. Biofizika 1973; 18: 235.

68. Ban FQ, Wetmore SD, Boyd RJ. J. Phys. Chem. A 1999; 103: 4303.

69. Pauwels E, Van Speybroeck V, Lahorte P, Waroquier M. J. Phys. Chem. A 2001; 105: 8794

70. Pauwels E, Van Speybroeck V, Waroquier M. Int. J. Quantum Chem. 2003; 91: 511.

71. Maseras F, Morokuma K. J. Comput. Chem. 1995; 15: 1170.

72. Svensson M, Humbel S, Froese RDJ, Matsubara T, Sieber S, Morokuma K. J. Phys. Chem. 1996; 100: 19357.

73. Humber S, Sieber S, Morokuma K. J. Chem. Phys. 1996; 105: 1959.

74. Matsubara T, Sieber S, Morokuma K. Int. J. Quantum Chem. 1996; 60: 1101.

75. Dapprich S, Komaromi I, Byun KS, Morokuma K, Frisch MJ. J. Mol. Struct. (Theochem) 1999; 462: 1.

76. Petrenko TL. J. Phys. Chem. A 2002; 106: 149.

77. Szyczewski A, Mobius K. J. Mol. Struct. 1994; 318: 87.

78. Szyczewski A. Appl. Radiat. Isot. 1996; 47: 1675.

79. Szyczewski A, Endeward B, Mobius K. Appl. Radiat. Isot. 1998; 49: 59. 